\title{
Two-loop operator matrix elements calculated up to finite terms
}

\author{
Y. Matiounine and J. Smith \\ Institute for Theoretical Physics, State University of New York at Stony Brook, Stony Brook, New York 11794-3840 \\ W. L. van Neerven \\ Instituut-Lorentz, University of Leiden, PO Box 9506, 2300 RA Leiden, The Netherlands
}

(Received 6 January 1998; published 23 April 1998)

\begin{abstract}
We present the two-loop corrected operator matrix elements calculated in $N$-dimensional regularization up to the finite terms which survive in the limit $\varepsilon=N-4 \rightarrow 0$. The anomalous dimensions of the local operators have been previously extracted from the pole terms and determine the scale evolution of the deep inelastic structure functions measured in unpolarized lepton hadron scattering. The finite $\varepsilon$-independent terms in the two-loop expressions are needed to renormalize the local operators up to third order in the strong coupling constant $\alpha_{s}$. Further the unrenormalized expressions for the two-loop corrected operator matrix elements can be inserted into specific one loop graphs to obtain a part of the third order contributions to these matrix elements. This work is a first step in obtaining the anomalous dimensions up to third order so that a complete next-to-next-to-leading order analysis can be carried out for deep inelastic electroproduction.
\end{abstract}

[S0556-2821(98)03211-1]

PACS number(s): 12.38.Bx, 11.15.Bt

\section{INTRODUCTION}

One of the most important successes of the theory of perturbative quantum chromodynamics $(\mathrm{QCD})$ is the prediction of the scale evolution of the structure functions $\mathrm{F}_{i}\left(x, Q^{2}\right)$ measured in deep-inelastic lepton-hadron scattering (for reviews see [1]). From these structure functions one can infer the parton densities, which serve as input for many other deep-inelastic (hard) processes and lead to a wealth of predictions for cross sections (for a review see [2]). Many of these predictions have been tested by now and are in very good agreement with the experimental data. The scale evolution of the structure functions is determined by the anomalous dimensions of local composite operators $O_{i}^{n}(x)$, $i=q, g$, where $n$ denotes the spin, which show up in the expansion of the product of two local electroweak currents $\mathrm{J}_{\mu}(x)$ and $\mathbf{J}_{\nu}(y)$ around the light cone $(x-y)^{2}=0$. The structure functions are proportional to the Fourier transform into momentum space of the product of these two currents sandwiched between hadronic states. In this way the structure functions can be written as a product of operator matrix elements (OME's) and coefficient functions. The former describe the long distance (low momenta) properties of QCD whereas the latter account for the short distance (large momenta) behavior of the specific quantities, such as e.g. the structure functions, under consideration. Both the anomalous dimensions and the coefficient functions are calculable order by order in perturbation theory so that they can be expressed in a series expansion in the strong coupling constant $\alpha_{s}$. The finite anomalous dimensions are responsible for the scale dependence of the structure functions and the parton densities. On the other hand the OME's themselves are of a nonperturbative nature, and, apart from some attempts using lattice gauge field theory, cannot yet be determined from first principles. Hence they have to be determined from models and fits to experimental data. In the past one has put a lot of effort into calculating the lowest order coefficients in the perturbation series for the anomalous dimensions and the coefficient functions. In [3,4] the order $\alpha_{s}$ contributions to the anomalous dimensions were obtained by evaluating the one-loop OME's which follow from inserting the local operators $O_{i}^{n}(x)$ between quark and gluon states (for an alternative method see [5]). The second order contributions, which involved the evaluation of two-loop OME's, have been computed by various groups [6-9]. As far as the coefficient functions are concerned they have been evaluated for many processes up to next-to-leading order (NLO) (for a recent review see [2]). Combining them with the above NLO anomalous dimensions one can make a complete NLO analysis of many quantities such as cross sections for Drell-Yan production or deep-inelastic structure functions.

The advent of the DESY ep collider HERA opened up a new era of deep-inelastic lepton-hadron experiments with much higher statistics than was previously available. Furthermore, the values of $x$ and $Q^{2}$, on which the structure functions depend, could be extended beyond those accessible by earlier fixed target experiments. This enables QCD to be tested with an even higher degree of precision so that effects beyond NLO can be studied. In particular both the small and the large $x$-regions have attracted much attention in the literature (for a review see e.g. [10]). Hence it will be necessary to extend the existing expressions for the anomalous dimensions and the coefficient functions beyond NLO. The first step to obtain the next-to-next-to-leading order (NNLO) corrections has been made in [11] where order $\alpha_{s}^{2}$ corrections to the coefficient functions for deep-inelastic leptonhadron scattering have been computed. The same program has also been completed for the Drell-Yan process in [12]. For a consistent NNLO analysis we also need the third order contributions to the anomalous dimensions. Unfortunately the latter are not known yet except for those corresponding to the operators $O_{i}^{n}(x)$ with spin $n=2,4,6,8$ and 10 . They have been computed in [13] using completely different meth- 
ods than those used in [6-9]. Using the above second order coefficient functions and the third order anomalous dimensions it is possible to make a NNLO analysis of the structure functions $F_{2}\left(x, Q^{2}\right)$ and $F_{3}\left(x, Q^{2}\right)$ as long as one limits oneself to the large $x \quad(x>0.01)$ and small $Q^{2}$ (see [14]). If one wants to study the behavior of the structure functions at small $x$ or at large $Q^{2}$, knowledge of the anomalous dimensions for any spin is indispensable. Since the full calculation of the latter quantities is a tremendous enterprise, it has to be carried out step by step. The first step is to compute the two-loop OME's up to finite terms which is an extension of the work done in [6-9]. These finite terms are needed to carry out the renormalization of the three-loop graphs since they determine the single pole terms in $\mathrm{N}$-dimensional regularization from which one has to extract the third order contributions to the anomalous dimensions. Moreover, by inserting the unrenormalized two-loop corrected OME's in oneloop graphs one gets parts of the expressions for the unrenormalized three-loop graphs. This method has been used previously to get parts of the two-loop expressions by inserting the one-loop corrected OME's into one-loop graphs.

In Sec. II we will give an outline of the calculation which will be carried out in the Feynman gauge. Although this gauge entails some complications due to the mixing of physical and unphysical operators, it is the only one in which it is feasible to perform a calculation of Feynman graphs for the OME's beyond two-loop order. All other gauges, such as the axial gauge used in [8], lead to even more complications, which we want to avoid. The long expressions obtained for the full OME's are presented in Appendix A while Appendix $B$ contains results for non-physical OME's, which are needed to carry out the renormalization program.

\section{CALCULATION OF THE TWO-LOOP OPERATOR MATRIX ELEMENTS}

In this section we will give an outline of the calculation of the OME's up to two-loop order. The operators, which appear in unpolarized lepton-hadron scattering, can be divided into singlet and non-singlet parts with respect to the flavor group. In leading twist (namely twist 2 ) the non-singlet quark operator of spin $n$ is given by

$$
\begin{aligned}
O_{q, k}^{\mu_{1}, \mu_{2} \cdots \mu_{n}}(x)= & \frac{1}{2} i^{n-1} \mathcal{S}\left[\bar{\psi}(x) \gamma^{\mu_{1}} D^{\mu_{2} \cdots} D^{\mu_{n}} \frac{\lambda_{k}}{2} \psi(x)\right. \\
& + \text { trace terms }] .
\end{aligned}
$$

In the singlet case there are two operators. The quark operator is represented by

$$
\begin{aligned}
O_{q}^{\mu_{1}, \mu_{2} \cdots \mu_{n}}(x)= & \frac{1}{2} i^{n-1} \mathcal{S}\left[\bar{\psi}(x) \gamma^{\mu_{1}} D^{\mu_{2} \cdots} D^{\mu_{n}} \psi(x)\right. \\
& + \text { trace terms }],
\end{aligned}
$$

and the gluon operator is given by

$$
\begin{aligned}
O_{g}^{\mu_{1}, \mu_{2} \cdots \mu_{n}}(x)= & \frac{1}{2} i^{n-2} \mathcal{S}\left[F_{a \alpha}^{\mu_{1}}(x) D^{\mu_{2} \cdots} D^{\mu_{n-1}} F_{a}^{\alpha \mu_{n}}(x)\right. \\
& + \text { trace terms }] .
\end{aligned}
$$

In these composite operators $\psi$ and $F_{a}^{\mu \nu}$ stand for the quark field and the gluon field tensor respectively. The $\lambda_{k}$ in Eq. (2.1) represent the generators of the flavor group and the index $a$ in Eq. (2.3) stands for the color. Further, the above operators are irreducible tensors with respect to the Lorentz group so that they have to be symmetric and traceless in all their Lorentz indices $\mu_{i}$. From the operators above one can derive the Feynman rules for the operator vertices in the standard way (see e.g. $[6,15,16]$ ). This derivation is facilitated if the operators are multiplied by the source

$$
J_{\mu_{1} \mu_{2} \cdots \mu_{n}}=\Delta_{\mu_{1}} \Delta_{\mu_{2}} \cdots \Delta_{\mu_{n}},
$$

with $\Delta^{2}=0$ in order to eliminate the trace terms in Eqs. (2.1)-(2.3). Hence all operator vertices in momentum space are multiplied by a factor $(\Delta \cdot p)^{n}$. For the computation of the OME's denoted by

$$
A_{i j}=\left\langle j(p)\left|O_{i}\right| j(p)\right\rangle
$$

with $i, j=q, g$ we choose the Feynman gauge except for the one-loop graphs for which we take the general covariant gauge. For this choice the gluon propagator equals

$$
\Delta_{a b}^{\mu \nu}(k)=\frac{i \delta_{a b}}{k^{2}}\left(-g^{\mu \nu}+(1-\xi) \frac{k^{\mu} k^{\nu}}{k^{2}}\right)
$$

The matrix element (2.5) has to be considered as a connected Green function with the external legs amputated but with the external self-energies of the partons $j$ included. In this paper all quarks and gluons are taken to be massless and the external momentum $p$ is off-shell $\left(p^{2}<0\right)$ in order to get finite expressions for the OME's. This choice implies that the OME's are not gauge invariant so that they cease to be ordinary S-matrix elements. Moreover, they acquire unphysical parts which can be split into two classes. The first class originates from the fact that the equations of motion (EOM) do not apply anymore, which is the case for both non-singlet and singlet OME's. The second class can be traced back to the mixing between so-called gauge invariant (GI) or physical (PHYS) and non-gauge invariant (NGI) operators which originate from the Yang-Mills (here gluonic) sector (see $[17,18,19,20])$. Therefore this second class of unphysical operators only shows up in the singlet case. The first class does not contribute to the operator renormalization constants in contrast to the second class which affects the aforementioned constants via the mixing between the GI and NGI operators as we will see below. The way to deal with this mixing is described in [15,16,21,22].

The calculation of the Feynman graphs corresponding to the physical operators of Eqs. (2.1)-(2.3), which are depicted in the figures in [6], proceeds in the standard way. The figures and definitions for the corresponding unphysical operators are given in [15]. The Feynman integrals reveal ultraviolet divergences which are regularized using the method of $N$-dimensional regularization. In this way the above divergences show up in the form of pole terms of the type $(1 / \varepsilon)^{k}$ 
with $\varepsilon=N-4$. In [6-9] it was sufficient to evaluate the oneloop graphs up to finite terms and the two-loop graphs up to single pole terms in order to get the second order anomalous dimensions. Here we have to include terms proportional to $\varepsilon$ in the one-loop expressions and the two-loop graphs have to be computed up to terms which are finite in the limit $\varepsilon \rightarrow 0$. The way to compute the two-loop Feynman integrals up to finite terms is presented in [15] and in Appendix B of [23]. We used the program FORM [24] to do the necessary algebra.

To check our results for the Feynman diagrams it is useful to have explicit expressions for the pole terms in $\varepsilon$. Therefore we will now present the OME's expressed in renormalization group coefficients, which are defined in $[11,12]$. The explicit formulas for the physical and unphysical OME's can be found in Appendixes A and B respectively. Further, it is implicitly understood that all quantities in the main text, in particular the anomalous dimensions $\gamma_{i j}(i, j=q, g)$, are Mellin transforms but to avoid additional indices we do not write a superscript $n$ to indicate this explicitly. [Another way to interpret the formulas is that the OME's are given in parton momentum fraction $(z)$ space when the anomalous dimensions are replaced by minus the corresponding AltarelliParisi splitting functions and the multiplications in moment space are replaced by convolutions.] We have written the OME's in such a way that all renormalization group coefficients appearing in the expressions below are renormalized in the modified minimal subtraction $(\overline{\mathrm{MS}})$ scheme. Up to order $\alpha_{s}^{2}$ the non-singlet and the singlet OME's can be decomposed as

$$
\hat{A}_{q q}^{r}=\left[\Delta \hat{A}_{q q}^{r, \mathrm{PHYS}}+p \frac{\Delta \cdot p}{p^{2}} \hat{A}_{q q}^{r, \mathrm{EOM}}\right](\Delta \cdot p)^{n-1},
$$

where $\hat{A}_{q q}^{r \text { PHYS }}$ and $\hat{A}_{q q}^{r \text {,EOM }}$ with $r=$ NS, S stand for the physical and unphysical parts respectively. The latter enter due to the breakdown of the equations of motion. The non-singlet OME can now be expressed in renormalization group coefficients as follows:

$$
\begin{aligned}
& \hat{A}_{q q}^{\mathrm{NS}, \mathrm{PHYS}}= 1+\hat{a}_{s} S_{\varepsilon}\left(\frac{-p^{2}}{\mu^{2}}\right)^{\varepsilon / 2}\left[\frac{1}{\varepsilon} \gamma_{q q}^{\mathrm{NS},(0)}+a_{q q}^{\mathrm{NS},(1)}\right. \\
&\left.+\varepsilon a_{q q}^{\mathrm{NS}, \varepsilon,(1)}\right]+\hat{a}_{s}^{2} S_{\varepsilon}^{2}\left(\frac{-p^{2}}{\mu^{2}}\right)^{\varepsilon}\left[\frac { 1 } { \varepsilon ^ { 2 } } \left\{\frac{1}{2}\left(\gamma_{q q}^{\mathrm{NS},(0)}\right)^{2}\right.\right. \\
&\left.-\beta_{0} \gamma_{q q}^{\mathrm{NS},(0)}\right\}+\frac{1}{\varepsilon}\left\{\frac{1}{2} \gamma_{q q}^{\mathrm{NS},(1)}-2 \beta_{0} a_{q q}^{\mathrm{NS},(1)}\right. \\
&\left.+\gamma_{q q}^{\mathrm{NS},(0)} a_{q q}^{\mathrm{NS},(1)}-\hat{\xi} \frac{d a_{q q}^{\mathrm{NS},(1)}}{d \hat{\xi}} z_{\xi}\right\}+a_{q q}^{\mathrm{NS},(2)} \\
&-2 \beta_{0} a_{q q}^{\mathrm{NS}, \varepsilon,(1)}+\gamma_{q q}^{\mathrm{NS},(0)} a_{q q}^{\mathrm{NS}, \varepsilon,(1)} \\
&-\hat{\xi} \frac{d a_{q q}^{\mathrm{NS}, \varepsilon,(1)}}{d \hat{\xi}} z_{\xi}, \\
& \hat{\xi}=1
\end{aligned}
$$

where $S_{\varepsilon}$ denotes the spherical factor given by

$$
S_{\varepsilon}=\exp \left[\frac{\varepsilon}{2}\left(\gamma_{E}-\ln 4 \pi\right)\right],
$$

which originates from $N$-dimensional regularization. In the expressions above all quantities that are unrenormalized with respect to operator, coupling constant $\alpha_{s}$ and gauge constant $\xi$ renormalization are indicated by a caret. The finite terms are written in such a way that after all renormalizations the non-logarithmic terms [with respect to $\ln \left(-p^{2} / \mu^{2}\right)$ ] become equal to $a_{q q}^{\mathrm{NS},(\mathrm{k})}(k=1,2)$. Further, we have introduced a shorthand notation for the strong coupling constant so that

$$
a_{s}=\frac{\alpha_{s}}{4 \pi}, \quad \alpha_{s}=\frac{g^{2}}{4 \pi} .
$$

The coefficients $\beta_{0}$ and $z_{\xi}$, which originate from coupling constant and gauge constant renormalization, are given by

$$
\begin{gathered}
\hat{a}_{s}=a_{s}\left[1+a_{s} S_{\varepsilon}\left(2 \beta_{0} \frac{1}{\varepsilon}\right)\right], \\
\hat{\xi}=\xi\left[1+a_{s} S_{\varepsilon}\left(z_{\xi} \frac{1}{\varepsilon}\right)\right],
\end{gathered}
$$

where

$$
\beta_{0}=\frac{11}{3} C_{A}-\frac{8}{3} n_{f} T_{f}, \quad z_{\xi}=C_{A}\left(-\frac{10}{3}-(1-\xi)\right)+\frac{8}{3} n_{f} T_{f} .
$$

In QCD $[S U(N)]$ the color factors are given by $C_{F}=\left(N^{2}\right.$ $-1) / 2 N, C_{A}=N, T_{f}=1 / 2$ and $n_{f}$ stands for the number of light flavors. Finally the $\gamma_{i j}^{(k)}$ denote the coefficients of the order $a_{s}^{k+1}$ terms appearing in the series expansions of the anomalous dimensions. Using the same notation we can also express the unphysical part of the non-singlet OME in Eq. (2.5) in the aforementioned renormalization group coefficients

$$
\begin{aligned}
\hat{A}_{q q}^{\mathrm{NS}, \mathrm{EOM}}= & \hat{a}_{s} S_{\varepsilon}\left(\frac{-p^{2}}{\mu^{2}}\right)^{\varepsilon / 2}\left[b_{q q}^{\mathrm{NS},(1)}+\varepsilon b_{q q}^{\mathrm{NS}, \varepsilon,(1)}\right] \\
& +\hat{a}_{s}^{2} S_{\varepsilon}^{2}\left(\frac{-p^{2}}{\mu^{2}}\right)^{\varepsilon}\left[\frac { 1 } { \varepsilon } \left\{\gamma_{q q}^{\mathrm{NS},(0)} b_{q q}^{\mathrm{NS},(1)}-2 \beta_{0} b_{q q}^{\mathrm{NS},(1)}\right.\right. \\
& \left.-\hat{\xi} \frac{d b_{q q}^{\mathrm{NS},(1)}}{d \hat{\xi}} z_{\xi}\right\}+b_{q q}^{\mathrm{NS},(2)}-2 \beta_{0} b_{q q}^{\mathrm{NS}, \varepsilon,(1)} \\
& \left.+\gamma_{q q}^{\mathrm{NS},(0)} b_{q q}^{\mathrm{NS}, \varepsilon,(1)}-\hat{\xi} \frac{d b_{q q}^{\mathrm{NS}, \varepsilon,(1)}}{d \hat{\xi}} z_{\xi}\right]_{\hat{\xi}=1} .
\end{aligned}
$$

Since the singlet OME is only computed up to second order, the unphysical part arises from the fact that the equations of motion are not satisfied. In this order there is no need to introduce NGI operators. The singlet OME can be split into nonsinglet (NS) and purely singlet (PS) parts

$$
A_{q q}^{\mathrm{S}}=A_{q q}^{\mathrm{NS}}+A_{q q}^{\mathrm{PS}},
$$


where the purely singlet physical OME is

$$
\begin{aligned}
\hat{A}_{q q}^{\mathrm{PS}, \mathrm{PHYS}}= & \hat{a}_{s}^{2} S_{\varepsilon}^{2}\left(\frac{-p^{2}}{\mu^{2}}\right)^{\varepsilon}\left[\frac{1}{\varepsilon^{2}}\left\{\frac{1}{2} \gamma_{q g}^{(0)} \gamma_{g q}^{(0)}\right\}+\frac{1}{\varepsilon}\left\{\frac{1}{2} \gamma_{q q}^{\mathrm{PS},(1)}\right.\right. \\
& \left.\left.+\gamma_{q g}^{(0)} a_{g q}^{(1)}\right\}+a_{q q}^{\mathrm{PS},(2)}+\gamma_{q g}^{(0)} a_{g q}^{\varepsilon,(1)}\right]
\end{aligned}
$$

and the purely singlet unphysical OME is

$$
\hat{A}_{q q}^{\mathrm{PS}, \mathrm{EOM}}=\hat{a}_{s}^{2} S_{\varepsilon}^{2}\left(\frac{-p^{2}}{\mu^{2}}\right)^{\varepsilon}\left[\frac{1}{\varepsilon}\left\{\gamma_{q g}^{(0)} b_{g q}^{(1)}\right\}+b_{q q}^{\mathrm{PS},(2)}+\gamma_{q g}^{(0)} b_{g q}^{\varepsilon,(1)}\right] .
$$

The mixing with NGI operators does occur in $\hat{A}_{q g, \mu \nu}$ which can be written as

$$
\hat{A}_{q g, \mu \nu}=\hat{A}_{q g}^{\mathrm{PHYS}} T_{\mu \nu}^{(1)}+\hat{A}_{q g}^{\mathrm{EOM}} T_{\mu \nu}^{(2)}+\hat{A}_{q g}^{\mathrm{NGI}} T_{\mu \nu}^{(3)} .
$$

In this expression the tensors are given by

$$
\begin{gathered}
T_{\mu \nu}^{(1)}=\frac{1+(-1)^{n}}{2}\left[g_{\mu \nu}-\frac{p_{\mu} \Delta_{\nu}+\Delta_{\mu} p_{\nu}}{\Delta \cdot p}+\frac{\Delta_{\mu} \Delta_{\nu} p^{2}}{(\Delta \cdot p)^{2}}\right](\Delta \cdot p)^{n}, \\
T_{\mu \nu}^{(2)}=\frac{1+(-1)^{n}}{2}\left[\frac{p_{\mu} p_{\nu}}{p^{2}}-\frac{p_{\mu} \Delta_{\nu}+\Delta_{\mu} p_{\nu}}{\Delta \cdot p}+\frac{\Delta_{\mu} \Delta_{\nu} p^{2}}{(\Delta \cdot p)^{2}}\right](\Delta \cdot p)^{n},
\end{gathered}
$$

and

$$
T_{\mu \nu}^{(3)}=\frac{1+(-1)^{n}}{2}\left[-\frac{p_{\mu} \Delta_{\nu}+\Delta_{\mu} p_{\nu}}{2 \Delta \cdot p}+\frac{\Delta_{\mu} \Delta_{\nu} p^{2}}{(\Delta \cdot p)^{2}}\right](\Delta \cdot p)^{n} .
$$

For later purposes we also define the tensor

$$
T_{\mu \nu}^{(4)}=\frac{1+(-1)^{n}}{2}\left[\frac{p_{\mu} \Delta_{\nu}+\Delta_{\mu} p_{\nu}}{2 \Delta \cdot p}\right](\Delta \cdot p)^{n} .
$$

The above tensors satisfy the following relations:

$$
\begin{gathered}
p^{\mu} T_{\mu \nu}^{(i)}=0 \quad(i=1,2), \quad p^{\mu} T_{\mu \nu}^{(i)} \neq 0 \quad(i=3,4), \\
p^{\mu} p^{\nu} T_{\mu \nu}^{(i)}=0 \quad(i=1,2,3), \quad p^{\mu} p^{\nu} T_{\mu \nu}^{(4)} \neq 0 .
\end{gathered}
$$

Using these relations one can show that the OME's in Eq. (2.18) satisfy the following Ward-identities (WIs) for $i=q$ :

$$
p^{\mu} \hat{A}_{i g, \mu \nu}=\frac{1+(-1)^{n}}{2}\left[-p_{\nu}+\frac{\Delta_{\nu} p^{2}}{\Delta \cdot p}\right](\Delta \cdot p)^{n} \hat{A}_{i g}^{\mathrm{NGI}},
$$

$$
p^{\mu} p^{\nu} \hat{A}_{i g, \mu \nu}=0
$$

The WI in Eq. (2.25) shows that the unphysical part in Eq. (2.18), given by $\hat{A}_{q g}^{\mathrm{NGI}}$, is due to the NGI operator $O_{A}$ in Eq. (B1). The second term in Eq. (2.18), given by $\hat{A}_{q g}^{\mathrm{EOM}}$, is also unphysical due to the fact that we cannot apply the equations of motion. The physical part of the OME in Eq. (2.18) becomes

$$
\begin{aligned}
\hat{A}_{q g}^{\mathrm{PHYS}}= & \hat{a}_{s} S_{\varepsilon}\left(\frac{-p^{2}}{\mu^{2}}\right)^{\varepsilon / 2}\left[\frac{1}{\varepsilon} \gamma_{q g}^{(0)}+a_{q g}^{(1)}+\varepsilon a_{q g}^{\varepsilon,(1)}\right]+\hat{a}_{s}^{2} S_{\varepsilon}^{2}\left(\frac{-p^{2}}{\mu^{2}}\right)^{\varepsilon}\left[\frac{1}{\varepsilon^{2}}\left\{\frac{1}{2}\left(\gamma_{q q}^{(0)} \gamma_{q g}^{(0)}+\gamma_{q g}^{(0)} \gamma_{g g}^{(0)}\right)-\beta_{0} \gamma_{q g}^{(0)}\right\}\right. \\
& +\frac{1}{\varepsilon}\left\{\frac{1}{2} \gamma_{q g}^{(1)}-2 \beta_{0} a_{q g}^{(1)}+\gamma_{q g}^{(0)} a_{g g}^{(1)}+a_{q g}^{(1)} \gamma_{q q}^{(0)}-\hat{\xi} \frac{d a_{q g}^{(1)}}{d \hat{\xi}} z_{\xi}\right\}+a_{q g}^{(2)}-2 \beta_{0} a_{q g}^{\varepsilon,(1)}+\gamma_{q g}^{(0)} a_{g g}^{\varepsilon,(1)} \\
& \left.+\gamma_{q q}^{(0)} a_{q g}^{\varepsilon,(1)}-\hat{\xi} \frac{d a_{q g}^{\varepsilon,(1)}}{d \hat{\xi}} z_{\xi}\right]_{\hat{\xi}=1} .
\end{aligned}
$$

The unphysical parts are given by

$$
\begin{aligned}
\hat{A}_{q g}^{\mathrm{EOM}}= & \hat{a}_{s} S_{\varepsilon}\left(\frac{-p^{2}}{\mu^{2}}\right)^{\varepsilon / 2}\left[b_{q g}^{(1)}+\varepsilon b_{q g}^{\varepsilon,(1)}\right]+\hat{a}_{s}^{2} S_{\varepsilon}^{2}\left(\frac{-p^{2}}{\mu^{2}}\right)^{\varepsilon}\left[\frac{1}{\varepsilon}\left\{\gamma_{q g}^{(0)} b_{g g}^{(1)}+\gamma_{q q}^{(0)} b_{q g}^{(1)}-2 \beta_{0} b_{q g}^{(1)}-\hat{\xi} \frac{d b_{q g}^{(1)}}{d \hat{\xi}} z_{\xi}\right\}\right. \\
& \left.+b_{q g}^{(2)}-2 \beta_{0} b_{q g}^{\varepsilon,(1)}+\gamma_{q g}^{(0)} b_{g g}^{\varepsilon,(1)}+\gamma_{q q}^{(0)} b_{q g}^{\varepsilon,(1)}-\hat{\xi} \frac{d b_{q g}^{\varepsilon,(1)}}{d \hat{\xi}} z_{\xi}\right]_{\hat{\xi}=1}
\end{aligned}
$$

and

$$
\hat{A}_{q g}^{\mathrm{NGI}}=\hat{a}_{s}^{2} S_{\varepsilon}^{2}\left(\frac{-p^{2}}{\mu^{2}}\right)^{\varepsilon}\left[\frac{1}{\varepsilon^{2}}\left\{\gamma_{q g}^{(0)} \gamma_{g A}^{(0)}\right\}+\frac{1}{\varepsilon}\left\{\gamma_{q g}^{(0)} a_{g A}^{(1)}\right\}+a_{q A}^{(2)}+\gamma_{q g}^{(0)} a_{g A}^{\varepsilon,(1)}\right]
$$


The coefficients with a subscript $A$ originate from the NGI operator $O_{A}$ whose matrix element will be presented below when we discuss the renormalization.

The next OME $\hat{A}_{g q}$ can be decomposed as

$$
\hat{A}_{g q}=\left[\Delta \hat{A}_{g q}^{\mathrm{PHYS}}+\not p \frac{\Delta \cdot p}{p^{2}} \hat{A}_{g q}^{\mathrm{EOM}}\right](\Delta \cdot p)^{n},
$$

where the last term again represents the unphysical part due to the breakdown of the equations of motion. The physical part equals

$$
\begin{aligned}
\hat{A}_{g q}^{\mathrm{PHYS}}= & \hat{a}_{s} S_{\varepsilon}\left(\frac{-p^{2}}{\mu^{2}}\right)^{\varepsilon / 2}\left[\frac{1}{\varepsilon} \gamma_{g q}^{(0)}+a_{g q}^{(1)}+\varepsilon a_{g q}^{\varepsilon,(1)}\right]+\hat{a}_{s}^{2} S_{\varepsilon}^{2}\left(\frac{-p^{2}}{\mu^{2}}\right)^{\varepsilon}\left[\frac{1}{\varepsilon^{2}}\left\{\frac{1}{2} \gamma_{g q}^{(0)}\left(\gamma_{g g}^{(0)}+\gamma_{q q}^{\mathrm{NS},(0)}\right)-\beta_{0} \gamma_{g q}^{(0)}\right\}\right. \\
& +\frac{1}{\varepsilon}\left\{\frac{1}{2} \gamma_{g q}^{(1)}-2 \beta_{0} a_{g q}^{(1)}+\gamma_{g q}^{(0)} a_{q q}^{\mathrm{NS},(1)}+\gamma_{g g}^{(0)} a_{g q}^{(1)}+\gamma_{g A}^{(0)}\left(a_{A q}^{(1)}+a_{B q}^{(1)}\right)-\hat{\xi} \frac{d a_{g q}^{(1)}}{d \hat{\xi}} z_{\xi}\right\}+a_{g q}^{(2)}-2 \beta_{0} a_{g q}^{\varepsilon,(1)} \\
& \left.+\gamma_{g q}^{(0)} a_{q q}^{\mathrm{NS}, \varepsilon,(1)}+\gamma_{g g}^{(1)} a_{g q}^{\varepsilon,(1)}+\gamma_{g A}^{(0)}\left(a_{A q}^{\varepsilon,(1)}+a_{B q}^{\varepsilon,(1)}\right)-\hat{\xi} \frac{d a_{g q}^{\varepsilon,(1)}}{d \hat{\xi}} z_{\xi}\right]_{\hat{\xi}=1} .
\end{aligned}
$$

The coefficients with the subscripts $A$ and $B$ refer to the NGI operators $O_{A}$ and $O_{B}$ presented in Eq. (B1) and Eq. (B5) respectively. The latter shows up for the first time in order $\alpha_{s}^{2}$ in the physical part of $\hat{A}_{g q}$. The corresponding OME's will be given below when we discuss the renormalization of the physical operators. The unphysical part of Eq. (2.30) equals

$$
\begin{aligned}
\hat{A}_{g q}^{\mathrm{EOM}}= & \hat{a}_{s} S_{\varepsilon}\left(\frac{-p^{2}}{\mu^{2}}\right)^{\varepsilon / 2}\left[b_{g q}^{(1)}+\varepsilon b_{g q}^{\varepsilon,(1)}\right]+\hat{a}_{s}^{2} S_{\varepsilon}^{2}\left(\frac{-p^{2}}{\mu^{2}}\right)^{\varepsilon}\left[\frac{1}{\varepsilon}\left\{\gamma_{g q}^{(0)} b_{q q}^{(1)}+\gamma_{g g}^{(0)} b_{g q}^{(1)}-2 \beta_{0} b_{g q}^{(1)}-\hat{\xi} \frac{d b_{g q}^{(1)}}{d \hat{\xi}} z_{\xi}\right\}+b_{g q}^{(2)}-2 \beta_{0} b_{g q}^{\varepsilon,(1)}\right. \\
& \left.+\gamma_{g q}^{(0)} b_{q q}^{\mathrm{NS}, \varepsilon,(1)}+\gamma_{g g}^{(0)} b_{g q}^{\varepsilon,(1)}-\hat{\xi} \frac{d b_{g q}^{\varepsilon,(1)}}{d \hat{\xi}} z_{\xi}\right]_{\hat{\xi}=1} \cdot
\end{aligned}
$$

The last OME $\hat{A}_{g g, \mu \nu}$ has a similar decomposition to the one presented in Eq. (2.18):

$$
\hat{A}_{g g, \mu \nu}=\hat{A}_{g g}^{\mathrm{PHYS}} T_{\mu \nu}^{(1)}+\hat{A}_{g g}^{\mathrm{EOM}} T_{\mu \nu}^{(2)}+\hat{A}_{g g}^{\mathrm{NGI}} T_{\mu \nu}^{(3)} .
$$

In particular it satisfies the same Ward identities as listed in Eq. (2.25) and Eq. (2.26) for $i=g$. Hence the second (unphysical) part in this expression originates from the breakdown of the equations of motion whereas the third term again originates from the NGI operator $O_{A}$ in Eq. (B1). The physical part of the OME is equal to

$$
\begin{aligned}
\hat{A}_{g g}^{\mathrm{PHYS}}= & 1+\hat{a}_{S} S_{\varepsilon}\left(\frac{-p^{2}}{\mu^{2}}\right)^{\varepsilon / 2}\left[\frac{1}{\varepsilon} \gamma_{g g}^{(0)}+a_{g g}^{(1)}+\varepsilon a_{g g}^{\varepsilon,(1)}\right]+\hat{a}_{s}^{2} S_{\varepsilon}^{2}\left(\frac{-p^{2}}{\mu^{2}}\right)^{\varepsilon}\left[\frac{1}{\varepsilon^{2}}\left\{\frac{1}{2}\left[\left(\gamma_{g g}^{(0)}\right)^{2}+\gamma_{g q}^{(0)} \gamma_{q g}^{(0)}\right]-\beta_{0} \gamma_{g g}^{(0)}\right\}\right. \\
& +\frac{1}{\varepsilon}\left\{\frac{1}{2} \gamma_{g g}^{(1)}-2 \beta_{0} a_{g g}^{(1)}+\gamma_{g g}^{(0)} a_{g g}^{(1)}+\gamma_{g q}^{(0)} a_{q g}^{(1)}+\gamma_{g A}^{(0)}\left(a_{A g}^{(1)}+a_{\omega g}^{(1)}\right)-\hat{\xi} \frac{d a_{g g}^{(1)}}{d \hat{\xi}} z_{\xi}\right\}+a_{g g}^{(2)}-2 \beta_{0} a_{g g}^{\varepsilon,(1)} \\
& \left.+\gamma_{g g}^{(0)} a_{g g}^{\varepsilon,(1)}+\gamma_{g q}^{(0)} a_{q g}^{\varepsilon,(1)}+\gamma_{g A}^{(0)}\left(a_{A g}^{\varepsilon,(1)}+a_{\omega g}^{\varepsilon,(1)}\right)-\hat{\xi} \frac{d a_{g g}^{\varepsilon,(1)}}{d \hat{\xi}} z_{\xi}\right]_{\hat{\xi}=1} .
\end{aligned}
$$

The unphysical parts are given by

$$
\begin{aligned}
\hat{A}_{g g}^{\mathrm{EOM}}= & \hat{a}_{S} S_{\varepsilon}\left(\frac{-p^{2}}{\mu^{2}}\right)^{\varepsilon / 2}\left[b_{g g}^{(1)}+\varepsilon b_{g g}^{\varepsilon,(1)}\right]+\hat{a}_{s}^{2} S_{\varepsilon}^{2}\left(\frac{-p^{2}}{\mu^{2}}\right)^{\varepsilon}\left[\frac{1}{\varepsilon}\left\{\gamma_{g g}^{(0)} b_{g g}^{(1)}-2 \beta_{0} b_{g g}^{(1)}+\gamma_{g A}^{(0)}\left(b_{A g}^{(1)}+b_{\omega g}^{(1)}\right)-\hat{\xi} \frac{d b_{g g}^{(1)}}{d \hat{\xi}} z_{\xi}\right\}\right. \\
& \left.+b_{g g}^{(2)}-2 \beta_{0} b_{g g}^{\varepsilon,(1)}+\gamma_{g A}^{(0)}\left(b_{A g}^{\varepsilon,(1)}+b_{\omega g}^{\varepsilon,(1)}\right)-\hat{\xi} \frac{d b_{g g}^{\varepsilon,(1)}}{d \hat{\xi}} z_{\xi}\right]_{\hat{\xi}=1}
\end{aligned}
$$

and 


$$
\begin{aligned}
\hat{A}_{g g}^{\mathrm{NGI}}= & \hat{a}_{s} S_{\varepsilon}\left(\frac{-p^{2}}{\mu^{2}}\right)^{\varepsilon / 2}\left[\frac{1}{\varepsilon} \gamma_{g A}^{(0)}+a_{g A}^{(1)}+\varepsilon a_{g A}^{\varepsilon,(1)}\right]+\hat{a}_{s}^{2} S_{\varepsilon}^{2}\left(\frac{-p^{2}}{\mu^{2}}\right)^{\varepsilon}\left[\frac{1}{\varepsilon^{2}}\left\{\frac{1}{2}\left(\gamma_{g g}^{(0)}+\gamma_{A g}^{(0)}+\gamma_{\omega g}^{(0)}-2 \beta_{0}\right) \gamma_{g A}^{(0)}\right\}\right. \\
& +\frac{1}{\varepsilon}\left\{\frac{1}{2} \gamma_{g A}^{(1)}-2 \beta_{0} a_{g A}^{(1)}+\gamma_{g g}^{(0)} a_{g A}^{(1)}+\gamma_{g A}^{(0)}\left(a_{A A}^{(1)}+a_{\omega A}^{(1)}\right)-\hat{\xi} \frac{d a_{g A}^{(1)}}{d \hat{\xi}} z_{\xi}\right\}+a_{g A}^{(2)}-2 \beta_{0} a_{g A}^{\varepsilon,(1)}+\gamma_{g g}^{(0)} a_{g A}^{\varepsilon,(1)} \\
& \left.+\gamma_{g A}^{(0)}\left(a_{A A}^{\varepsilon,(1)}+a_{\omega A}^{\varepsilon,(1)}\right)-\hat{\xi} \frac{d a_{g A}^{\varepsilon,(1)}}{d \hat{\xi}} z_{\xi}\right]_{\hat{\xi}=1},
\end{aligned}
$$

respectively. In these expressions we observe that there are quantities with the subscript $A$ and with the subscript $\omega$. The latter originate from the NGI ghost operator $O_{\omega}$ presented in Eq. (B2). The renormalization of the above OME's involves the mixing of the NGI (non-gauge-invariant) operators $O_{A}$, $O_{B}$ and $O_{\omega}$ mentioned above with the physical (gauge invariant) operators in Eqs. (2.1)-(2.3). Therefore we have to compute the matrix elements in Eq. (2.5) where the physical operators indicated by $i=q, g$ are replaced by the NGI ones labelled by $i=A, B, \omega$. To get the physical OME's which are finite up to order $\alpha_{s}^{2}$ the unphysical ones have fortunately only to be calculated up to order $\alpha_{s}$.

The operator renormalization proceeds as follows. First we have to perform coupling constant and gauge constant renormalization. This is achieved by replacing the bare constants by the renormalized ones by substituting Eqs. (2.11) and (2.12) in the above expressions for the OME's. Subsequently the OME's have to be multiplied by the operator renormalization constants to remove the remaining ultraviolet divergences. The most simple case is the renormalization of the non-singlet OME's since here we do not have mixing with physical and NGI operators. This renormalization is achieved by

$$
A_{q q}^{\mathrm{NS}, i}=\left(Z^{-1}\right)_{q q}^{\mathrm{NS}} \hat{A}_{q q}^{\mathrm{NS}, i},
$$

with $i=$ PHYS and EOM. The inverse of the operator renormalization constant equals

$$
\begin{aligned}
\left(Z^{-1}\right)_{q q}^{\mathrm{NS}}= & 1+a_{s} S_{\varepsilon}\left[-\frac{1}{\varepsilon} \gamma_{q q}^{\mathrm{NS},(0)}\right]+a_{s}^{2} S_{\varepsilon}^{2}\left[\frac { 1 } { \varepsilon ^ { 2 } } \left\{\frac{1}{2}\left(\gamma_{q q}^{\mathrm{NS},(0)}\right)^{2}\right.\right. \\
& \left.\left.-\beta_{0} \gamma_{q q}^{\mathrm{NS},(0)}\right\}-\frac{1}{2 \varepsilon} \gamma_{q q}^{\mathrm{NS},(1)}\right] .
\end{aligned}
$$

In the case of the singlet operators $A_{q q}^{\mathrm{S}, i}$ there is no mixing between GI and NGI operators (at least up to order $\alpha_{s}^{2}$ ). The singlet OME's are finite for $i=$ PHYS and EOM when

$$
A_{q q}^{\mathrm{S}, i}=\left(Z^{-1}\right)_{q q}^{\mathrm{S}} \hat{A}_{q q}^{\mathrm{S}, i}+\left(Z^{-1}\right)_{q g} \hat{A}_{g q}^{i},
$$

and the renormalization involves the matrix $Z_{i j}$ due to mixing with unphysical operators. Hence we have to invert this matrix in order to perform the operator renormalization. The first renormalization constant in Eq. (2.39) can be split as follows:

$$
\left(Z^{-1}\right)_{q q}^{\mathrm{S}}=\left(Z^{-1}\right)_{q q}^{\mathrm{NS}}+\left(Z^{-1}\right)_{q q}^{\mathrm{PS}},
$$

where the first part is equal to the expression (2.38) and the last part equals

$$
\left(Z^{-1}\right)_{q q}^{\mathrm{PS}}=a_{s}^{2} S_{\varepsilon}^{2}\left[\frac{1}{\varepsilon^{2}}\left\{\frac{1}{2} \gamma_{q g}^{(0)} \gamma_{g q}^{(0)}\right\}-\frac{1}{2 \varepsilon} \gamma_{q q}^{\mathrm{PS},(1)}\right] .
$$

The constant $\left(Z^{-1}\right)_{q g}$ in Eq. (2.39) also shows up in the renormalization of the next OME, namely

$$
A_{q g}^{i}=\left(Z^{-1}\right)_{q q}^{\mathrm{S}} \hat{A}_{q g}^{i}+\left(Z^{-1}\right)_{q g} \hat{A}_{g g}^{i},
$$

for $i=$ PHYS, EOM and NGI. It can be decomposed into renormalization group coefficients as follows:

$$
\begin{aligned}
\left(Z^{-1}\right)_{q g}= & a_{s} S_{\varepsilon}\left[-\frac{1}{\varepsilon} \gamma_{q g}^{(0)}\right]+a_{s}^{2} S_{\varepsilon}^{2}\left[\frac { 1 } { \varepsilon ^ { 2 } } \left\{\frac{1}{2} \gamma_{q g}^{(0)}\left(\gamma_{q q}^{(0)}+\gamma_{g g}^{(0)}\right)\right.\right. \\
& \left.\left.-\beta_{0} \gamma_{q g}^{(0)}\right\}-\frac{1}{2 \varepsilon} \gamma_{q g}^{(1)}\right] .
\end{aligned}
$$

For the renormalization of $A_{g q}^{\mathrm{PHYS}}$ in Eq. (2.31) and $A_{g q}^{\mathrm{EOM}}$ in Eq. (2.32) we also need the contributions of the NGI OME's. Here the finite expressions are given by

$$
A_{g q}^{i}=\left(Z^{-1}\right)_{g g}\left[\hat{A}_{g q}^{i}+\eta\left(\hat{A}_{A q}^{i}+\hat{A}_{B q}^{i}\right)\right]+\left(Z^{-1}\right)_{g q} \hat{A}_{q q}^{\mathrm{S}, i},
$$

where $\eta$ is defined as a source multiplying the NGI operators in the effective action [see Eq. (2.8) in [15]]. Here we will write this quantity as follows:

$$
\begin{aligned}
\eta= & a_{s} S_{\varepsilon}\left[-\frac{1}{\varepsilon} \gamma_{g A}^{(0)}\right]+a_{s}^{2} S_{\varepsilon}^{2}\left[\frac { 1 } { \varepsilon ^ { 2 } } \left\{\frac { 1 } { 2 } \left(\gamma_{A A}^{(0)}+\gamma_{\omega A}^{(0)}\right.\right.\right. \\
& \left.\left.\left.-\gamma_{g g}^{(0)}-2 \beta_{0}\right) \gamma_{g A}^{(0)}\right\}-\frac{1}{2 \varepsilon} \gamma_{g A}^{(1)}\right] .
\end{aligned}
$$

If we sandwich the operator $O_{A}$ between quark states [see Eq. (2.5)], we get

$$
\hat{A}_{A q}^{\mathrm{PHYS}}=\hat{a}_{S} S_{\varepsilon}\left(\frac{-p^{2}}{\mu^{2}}\right)^{\varepsilon / 2}\left[\frac{1}{\varepsilon} \gamma_{A q}^{(0)}+a_{A q}^{(1)}+\varepsilon a_{A q}^{\varepsilon,(1)}\right]
$$

and

$$
\hat{A}_{A q}^{\mathrm{EOM}}=\hat{a}_{S} S_{\varepsilon}\left(\frac{-p^{2}}{\mu^{2}}\right)^{\varepsilon / 2}\left[b_{A q}^{(1)}+\varepsilon b_{A q}^{\varepsilon,(1)}\right],
$$


up to lowest order. We proceed in a similar way for the operator $O_{B}$ and the OME's read

$$
\hat{A}_{B q}^{\mathrm{PHYS}}=\hat{a}_{s} S_{\varepsilon}\left(\frac{-p^{2}}{\mu^{2}}\right)^{\varepsilon / 2}\left[-\frac{1}{\varepsilon} \gamma_{A q}^{(0)}+a_{B q}^{(1)}+\varepsilon a_{B q}^{\varepsilon,(1)}\right]
$$

and

$$
\hat{A}_{B q}^{\mathrm{EOM}}=\hat{a}_{s} S_{\varepsilon}\left(\frac{-p^{2}}{\mu^{2}}\right)^{\varepsilon / 2}\left[b_{B q}^{(1)}+\varepsilon b_{B q}^{\varepsilon,(1)}\right] .
$$

Notice that $\hat{A}_{A q}$ and $\hat{A}_{B q}$ can be decomposed in a similar way to $\hat{A}_{g q}$ in Eq. (2.30). The operator renormalization constant appearing in the last part of Eq. (2.44) can now be written as

$$
\begin{aligned}
\left(Z^{-1}\right)_{g q}= & a_{s} S_{\varepsilon}\left[-\frac{1}{\varepsilon} \gamma_{g q}^{(0)}\right]+a_{s}^{2} S_{\varepsilon}^{2}\left[\frac { 1 } { \varepsilon ^ { 2 } } \left\{\frac{1}{2} \gamma_{g q}^{(0)}\left(\gamma_{g g}^{(0)}+\gamma_{q q}^{(0)}\right)\right.\right. \\
& \left.\left.-\beta_{0} \gamma_{g q}^{(0)}\right\}-\frac{1}{2 \varepsilon} \gamma_{g q}^{(1)}\right] .
\end{aligned}
$$

The first constant $\left(Z^{-1}\right)_{g g}$ in Eq. (2.44) also shows up in the renormalization of the OME:

$$
A_{g g}^{i}=\left(Z^{-1}\right)_{g q} \hat{A}_{q g}^{i}+\left(Z^{-1}\right)_{g g}\left[\hat{A}_{g g}^{i}+\eta\left(\hat{A}_{A g}^{i}+\hat{A}_{\omega g}^{i}\right)\right],
$$

for $i=$ PHYS, EOM and NGI. If we express this constant in the renormalization group coefficients,

$$
\begin{aligned}
\left(Z^{-1}\right)_{g g}= & 1+a_{s} S_{\varepsilon}\left[-\frac{1}{\varepsilon} \gamma_{g g}^{(0)}\right]+a_{s}^{2} S_{\varepsilon}^{2}\left[\frac { 1 } { \varepsilon ^ { 2 } } \left\{\frac{1}{2}\left(\gamma_{g g}^{(0)}\right)^{2}\right.\right. \\
& \left.\left.+\frac{1}{2} \gamma_{g q}^{(0)} \gamma_{q g}^{(0)}-\beta_{0} \gamma_{g g}^{(0)}\right\}-\frac{1}{2 \varepsilon} \gamma_{g g}^{(1)}\right],
\end{aligned}
$$

we obtain the finite physical (PHYS) and unphysical parts (EOM, NGI) of expression (2.51). In order to obtain these finite parts one has first to calculate the OME's which emerge when the NGI operators $O_{A}$ and $O_{\omega}$ are sandwiched between gluon states [see Eq. (2.5)]. They can be decomposed as follows:

$$
\hat{A}_{A g, \mu \nu}=\hat{A}_{A g}^{\mathrm{PHYS}} T_{\mu \nu}^{(1)}+\hat{A}_{A g}^{\mathrm{EOM}} T_{\mu \nu}^{(2)}+\hat{A}_{A g}^{\mathrm{NGI}} T_{\mu \nu}^{(3)}+\hat{A}_{A g}^{\mathrm{WI}} T_{\mu \nu}^{(4)},
$$

with

$$
\begin{gathered}
\hat{A}_{A g}^{\mathrm{PHYS}}=\hat{a}_{s} S_{\varepsilon}\left(\frac{-p^{2}}{\mu^{2}}\right)^{\varepsilon / 2}\left[\frac{1}{\varepsilon} \gamma_{A g}^{(0)}+a_{A g}^{(1)}+\varepsilon a_{A g}^{\varepsilon,(1)}\right], \\
\hat{A}_{A g}^{\mathrm{EOM}}=\hat{a}_{s} S_{\varepsilon}\left(\frac{-p^{2}}{\mu^{2}}\right)^{\varepsilon / 2}\left[b_{A g}^{(1)}+\varepsilon b_{A g}^{\varepsilon,(1)}\right], \\
\hat{A}_{A g}^{\mathrm{NGI}}=1+\hat{a}_{s} S_{\varepsilon}\left(\frac{-p^{2}}{\mu^{2}}\right)^{\varepsilon / 2}\left[\frac{1}{\varepsilon} \gamma_{A A}^{(0)}+a_{A A}^{(1)}+\varepsilon a_{A A}^{\varepsilon,(1)}\right],
\end{gathered}
$$

and

$$
\hat{A}_{A g}^{\mathrm{WI}}=\hat{a}_{S} S_{\varepsilon}\left(\frac{-p^{2}}{\mu^{2}}\right)^{\varepsilon / 2}\left[c_{A g}^{(1)}+\varepsilon c_{A g}^{\varepsilon,(1)}\right] .
$$

Similar algebraic expressions are obtained for $O_{\omega}$. They are given by

$$
\hat{A}_{\omega g, \mu \nu}=\hat{A}_{\omega g}^{\mathrm{PHYS}} T_{\mu \nu}^{(1)}+\hat{A}_{\omega g}^{\mathrm{EOM}} T_{\mu \nu}^{(2)}+\hat{A}_{\omega g}^{\mathrm{NGI}} T_{\mu \nu}^{(3)}+\hat{A}_{\omega g}^{\mathrm{WI}} T_{\mu \nu}^{(4)},
$$

with

$$
\begin{gathered}
\hat{A}_{\omega g}^{\mathrm{PHYS}}=\hat{a}_{s} S_{\varepsilon}\left(\frac{-p^{2}}{\mu^{2}}\right)^{\varepsilon / 2}\left[-\frac{1}{\varepsilon} \gamma_{A g}^{(0)}+a_{\omega g}^{(1)}+\varepsilon a_{\omega g}^{\varepsilon,(1)}\right], \\
\hat{A}_{\omega g}^{\mathrm{EOM}}=\hat{a}_{s} S_{\varepsilon}\left(\frac{-p^{2}}{\mu^{2}}\right)^{\varepsilon / 2}\left[b_{\omega g}^{(1)}+\varepsilon b_{\omega g}^{\varepsilon,(1)}\right], \\
\hat{A}_{\omega g}^{\mathrm{NGI}}=\hat{a}_{s} S_{\varepsilon}\left(\frac{-p^{2}}{\mu^{2}}\right)^{\varepsilon / 2}\left[\frac{1}{\varepsilon} \gamma_{\omega A}^{(0)}+a_{\omega A}^{(1)}+\varepsilon a_{\omega A}^{\varepsilon,(1)}\right],
\end{gathered}
$$

and

$$
\hat{A}_{\omega g}^{\mathrm{WI}}=\hat{a}_{S} S_{\varepsilon}\left(\frac{-p^{2}}{\mu^{2}}\right)^{\varepsilon / 2}\left[-c_{A g}^{(1)}-\varepsilon c_{A g}^{\varepsilon,(1)}\right]
$$

Notice that neither $\hat{A}_{A g, \mu \nu}$ in Eq. (2.54) nor $\hat{A}_{\omega g, \mu \nu}$ in Eq. (2.59) satisfy the (WIs) in Eq. (2.25) and Eq. (2.26). However, if we add them according to Eq. (2.51), the terms proportional to the tensor $T_{\mu \nu}^{(4)}$ cancel and the Ward identies are restored.

From the residues of the single pole terms appearing in the physical operator renormalization constants $Z_{i j}$ with $i, j=q, g$ given above one can now read off the anomalous dimensions presented in the $\overline{\mathrm{MS}}$ scheme. Here we agree with the results published in [8] (see also [15]). Therefore we have a check that the residues of the single and double pole terms are correct. By comparison with the algebraic expressions given above one can obtain all renormalization group coefficients such as $a_{i j}^{(k)}, a_{i j}^{\varepsilon,(1)}$, etc. Explicit expressions for the physical and unphysical OME's can be found in Appendixes $\mathrm{A}$ and $\mathrm{B}$ respectively. They contain all the finite second order terms which survive in the limit $\varepsilon \rightarrow 0$. These terms have not been calculated previously.

Note that a remarkable property is found for the nonsinglet OME $\hat{A}_{q q}^{\mathrm{NS}, \mathrm{PHYS}}$ in Eq. (2.8) for which the full expression is given in Eq. (A3). The first moment of this OME is equal to unity up to second order in $\alpha$ s provided we choose the Feynman gauge $(\hat{\xi}=1)$. This result is expected for the on-shell expression as it is a check of the Adler sum rule [25]. However the fact that all the coefficients of the terms in $\left(-p^{2} / \mu^{2}\right)$ are zero shows that the sum rule is true up to order $\alpha_{s}^{2}$ for the off-shell expression which is not an S-matrix element. Finally we want to comment on the use of the unrenormalized expressions given in Appendix A for the computation of the three-loop OME's. Since the external quark and gluon legs are off-shell one can insert our results into the Feynman integrals for one-loop graphs. In this way one gets expressions corresponding to some of the three-loop graphs. It is, however, clear that the most difficult Feynman integrals 
belonging to the non-planar diagrams, where all quark or gluon lines cross over, remain to be done.

\section{ACKNOWLEDGMENTS}

This research was supported in part by the National Science Foundation grant PHY-9722101.

\section{APPENDIX A}

In this appendix we present full expressions for the twoloop corrected operator matrix elements computed from the Feynman diagrams depicted in [6]. The second order contributions are calculated up to finite terms which survive in the limit $\varepsilon \rightarrow 0$. The OME's presented here are unrenormalized and external self-energy corrections are included. In these expressions definitions of the Riemann zeta-functions $\zeta(n)$ and the polylogarithms $\operatorname{Li}_{n}(z), \mathrm{S}_{n, m}(z)$ can be found in [26].
Also the distributions $(1 /(1-z))_{+}$and $(\ln (1-z) /(1-z))_{+}$are written simply as $1 /(1-z)$ and $\ln (1-z) /(1-z)$ respectively to shorten the formulas. Note that the OME's given in the text are the moments of the functions listed here, and so

$$
A_{i j}^{n}=\int_{0}^{1} d z z^{n-1} A_{i j}\left(z, \frac{-p^{2}}{\mu^{2}}, \frac{1}{\varepsilon}\right),
$$

where for simplicity we have not written the moment index $n$ on the functions. Also to simplify the expressions we define the phase-space factor

$$
F=\frac{\hat{\alpha}_{s}}{4 \pi} S_{\varepsilon}\left(\frac{-p^{2}}{\mu^{2}}\right)^{\varepsilon / 2} .
$$

We first split $\hat{A}_{q q}^{\mathrm{NS}}$ into physical and unphysical parts following the notation in Eq. (2.7). The physical part is

$$
\begin{aligned}
& \hat{A}_{q q}^{\mathrm{NS}, \mathrm{PHYS}}\left(z, \frac{-p^{2}}{\mu^{2}}, \frac{1}{\varepsilon}\right) \\
& =\delta(1-z)+F C_{F}\left(\frac{1}{\varepsilon}\left(-4-4 z+\frac{8}{1-z}+6 \delta(1-z)\right)+4-6 z+2\left(\frac{2}{1-z}-1-z\right)[\ln z+\ln (1-z)]-\delta(1-z)[7-4 \zeta(2)]\right. \\
& +\frac{1-\hat{\xi}}{1-z}+\varepsilon\left\{1-2 z+(2-3 z)[\ln z+\ln (1-z)]+\frac{1}{2}\left(\frac{2}{1-z}-1-z\right)\left[\zeta(2)+\{\ln z+\ln (1-z)\}^{2}\right]+\delta(1-z)\left[7-\frac{3}{4} \zeta(2)\right.\right. \\
& \left.\left.-4 \zeta(3)]+\frac{1}{2}(1-\hat{\xi})\left[2-\frac{1}{1-z}+\frac{\ln (1-z)}{1-z}+\frac{\ln z}{1-z}+\delta(1-z)\{-2+\zeta(2)\}\right]\right\}\right)+F^{2}\left(\frac { 1 } { \varepsilon ^ { 2 } } \left\{C _ { F } ^ { 2 } \left[40+8 z-\frac{48}{1-z}\right.\right.\right. \\
& \left.-2 \delta(1-z)[9-16 \zeta(2)]+32 \ln (1-z)\left(1+z-\frac{2}{1-z}\right)-8 \ln z\left(3+3 z-\frac{4}{1-z}\right)\right]+C_{A} C_{F}\left[\frac{44}{3}\left(1+z-\frac{2}{1-z}\right)\right. \\
& \left.-22 \delta(1-z)]+n_{f} C_{F} T_{f}\left[-\frac{16}{3}\left(1+z-\frac{2}{1-z}\right)+8 \delta(1-z)\right]\right\}+\frac{1}{\varepsilon}\left\{C _ { F } ^ { 2 } \left[-40-4 z+\frac{56}{1-z}+\delta(1-z)\left(\frac{87}{2}-36 \zeta(2)\right.\right.\right. \\
& -8 \zeta(3))-4 \ln (1-z)\left(1-11 z+\frac{6}{1-z}\right)+24 \ln ^{2}(1-z)\left(1+z-\frac{2}{1-z}\right)+4 \ln z\left(9-5 z-\frac{9}{1-z}\right) \\
& \left.-16 \ln z \ln (1-z) \frac{1}{1-z}-2 \ln ^{2} z\left(7+7 z-\frac{8}{1-z}\right)-8 \operatorname{Li}_{2}(1-z)(1+z)\right]+C_{A} C_{F}\left[-\frac{158}{9}+\frac{22}{9} z+\frac{238}{9} \frac{1}{1-z}\right. \\
& +\delta(1-z)\left(\frac{325}{6}-\frac{44}{3} \zeta(2)-12 \zeta(3)\right)+4\left(1+z-\frac{2}{1-z}\right)\left(\zeta(2)+\frac{11}{3} \ln (1-z)-\frac{1}{2} \ln 2 z\right)+\ln z\left(\frac{34}{3}+\frac{34}{3} z\right. \\
& \left.\left.\left.-\frac{44}{3} \frac{1}{1-z}\right)\right]+n_{f} C_{F} T_{f}\left[\frac{88}{9}-\frac{56}{9} z-\frac{56}{9} \frac{1}{1-z}+\delta(1-z)\left(-\frac{58}{3}+\frac{16}{3} \zeta(2)\right)-\frac{8}{3}\{\ln z+2 \ln (1-z)\}\left(1+z-\frac{2}{1-z}\right)\right]\right\} \\
& +C_{F}^{2}\left[\frac{188}{3}+\frac{4}{3} z-\frac{56}{1-z}+\zeta(2)\left(20-4 z+\frac{32}{3} z^{2}-\frac{4}{1-z}\right)+24 \zeta(3)\left(1-z+2 z^{2}-\frac{1}{1-z}\right)+\delta(1-z)\left(-\frac{541}{8}+\frac{97}{2} \zeta(2)\right.\right. \\
& \left.+54 \zeta(3)-\frac{74}{5} \zeta(2)^{2}\right)+\ln (1-z)\left(-38+38 z+\frac{28}{1-z}\right)+8 \ln (1-z) \zeta(2)\left(1+z-2 z^{2}-\frac{1}{1-z}\right) \\
& -\ln ^{2}(1-z)\left(13-31 z+\frac{6}{1-z}\right)+\frac{28}{3} \ln ^{3}(1-z)\left(1+z-\frac{2}{1-z}\right)-4 \ln (1-z) \operatorname{Li}_{2}(1-z)\left(3-z+4 z^{2}\right) \\
& -\ln z\left(\frac{68}{3}+\frac{68}{3} z-\frac{44}{1-z}\right)+\ln z \zeta(2)\left(2-14 z+16 z^{2}\right)-2 \ln z \ln (1-z)\left(3-17 z+\frac{10}{1-z}\right)
\end{aligned}
$$


$+2 \ln z \ln ^{2}(1-z)\left(3+3 z-\frac{10}{1-z}\right)+\ln ^{2} z\left(11-23 z-\frac{16}{3} z^{2}-\frac{15}{1-z}\right)-\ln ^{2} z \ln (1-z)\left(6-2 z+8 z^{2}+\frac{4}{1-z}\right)$

$-\ln ^{3} z\left(5+5 z-\frac{16}{3} \frac{1}{1-z}\right)-8 \ln z \operatorname{Li}_{2}(1-z)\left(1-3 z+4 z^{2}+\frac{2}{1-z}\right)-4 \operatorname{Li}_{2}(1-z)\left(6-12 z+\frac{1}{1-z}\right)$

$+4 \mathrm{Li}_{3}(1-z)\left(5-7 z+12 z^{2}\right)+\frac{8}{3}\left(9+\frac{1}{z}+12 z+4 z^{2}\right)\left[\ln z \ln (1+z)+\mathrm{Li}_{2}(-z)\right]-16\left[\ln z \operatorname{Li}_{2}(-z)\right.$

$\left.\left.-2 \mathrm{Li}_{3}(-z)\right]\left(1-\frac{1}{1-z}\right)-8 \mathrm{~S}_{12}(1-z)\left(1-7 z+6 z^{2}+\frac{4}{1-z}\right)\right]+C_{A} C_{F}\left[\frac{1}{27}\left(941-580 z-\frac{670}{1-z}\right)\right.$

$-\frac{1}{3} \zeta(2)\left(1-17 z+16 z^{2}+\frac{28}{1-z}\right)-2 \zeta(3)\left(10+4 z+12 z^{2}-\frac{17}{1-z}\right)+\delta(1-z)\left(-\frac{7081}{72}+\frac{301}{18} \zeta(2)\right.$

$\left.+28 \zeta(3)+\frac{49}{5} \zeta(2)^{2}\right)-\frac{2}{9} \ln (1-z)\left(61+16 z-\frac{119}{1-z}\right)+2 \ln (1-z) \zeta(2)\left(1+3 z+4 z^{2}-\frac{5}{1-z}\right)$

$+\left\{\frac{22}{3} \ln ^{2}(1-z)-\ln ^{3} z\right\}\left(1+z-\frac{2}{1-z}\right)-2 \ln z \zeta(2)\left(3+z+4 z^{2}-\frac{5}{1-z}\right)$

$+\frac{1}{6} \ln ^{2} z\left(47+47 z+16 z^{2}-\frac{22}{1-z}\right)+\frac{2}{3} \ln z \ln (1-z)\left(14+5 z-\frac{19}{1-z}\right)+\frac{1}{9} \ln z\left(14+176 z+\frac{101}{1-z}\right)$

$+4 \ln z \operatorname{Li}_{2}(1-z)\left(2+4 z^{2}-\frac{3}{1-z}\right)-4 \operatorname{Li}_{2}(1-z)\left(3 z-\frac{1}{1-z}\right)-4\left(3+\frac{1}{3 z}+4 z+\frac{4}{3} z^{2}\right)[\ln z \ln (1+z)$

$\left.+\mathrm{Li}_{2}(-z)\right]+\left(1-z+4 z^{2}-\frac{1}{1-z}\right)\left[\ln ^{2} z \ln (1-z)+2 \ln (1-z) \mathrm{Li}_{2}(1-z)-6 \operatorname{Li}_{3}(1-z)\right]+8\left[\ln z \operatorname{Li}_{2}(-z)\right.$

$\left.\left.-2 \mathrm{Li}_{3}(-z)\right]\left(1-\frac{1}{1-z}\right)+\mathrm{S}_{12}(1-z)\left(14-6 z+24 z^{2}-\frac{14}{1-z}\right)\right]+n_{f} C_{F} T_{f}\left[\frac{4}{27}\left(-22+5 z+\frac{32}{1-z}\right)\right.$

$+\delta(1-z)\left(\frac{569}{18}-\frac{46}{9} \zeta(2)-8 \zeta(3)\right)+\frac{4}{9}\{2 \ln (1-z)+\ln z\}\left(11-7 z-\frac{7}{1-z}\right)-\frac{2}{3}[2 \zeta(2)$

$\left.\left.+\{2 \ln (1-z)+\ln z\}^{2}\right]\left(1+z-\frac{2}{1-z}\right)\right]-2(-1)^{n}\left(C_{F}^{2}-\frac{1}{2} C_{A} C_{F}\right)\left\{\frac{1}{\varepsilon}[-8+8 z-4 \ln z(1+z)\right.$

$\left.+2\left(-1+z+\frac{2}{1+z}\right)\left[2 \zeta(2)+4 \ln z \ln (1+z)-\ln ^{2} z+4 \mathrm{Li}_{2}(-z)\right]\right]-\frac{41}{3}(1-z)-8 \ln (1-z)(1-z)$

$+2 \zeta(2)\left(3-3 z+\frac{8}{3} z^{2}\right)+8\left(z+\frac{1}{1+z}\right) \ln (1+z)\left[\zeta(2)+\ln z \ln (1+z)+2 \mathrm{Li}_{2}(-z)\right]-4(1+z)[\ln z \ln (1-z)$

$\left.+\mathrm{Li}_{2}(1-z)+\mathrm{Li}_{3}(-z)\right]-\frac{1}{3} \ln z(37+25 z)-\ln ^{2} z\left(6+\frac{8}{3} z^{2}\right)-2 \ln ^{2} z \ln (1+z)\left(3-z-\frac{4}{1+z}\right)$

$-2 \zeta(3)\left(3+z-\frac{2}{1+z}\right)+\frac{4}{3}\left(\frac{1}{z}+3 z+4 z^{2}\right)\left[\ln z \ln (1+z)+\operatorname{Li}_{2}(-z)+8 \mathrm{~S}_{12}(-z)\left(-1+3 z+\frac{4}{1+z}\right)\right.$

$+4\left(1-z-\frac{2}{1+z}\right)\left(\frac{1}{4} \ln ^{3} z-\ln z \ln ^{2}(1-z)-2 \ln z \ln (1-z) \ln (1+z)+\frac{1}{2} \ln ^{2} z \ln (1-z)-2 \ln (1-z) \operatorname{Li}_{2}(1-z)\right.$

$\left.\left.\left.-2 \ln (1+z) \mathrm{Li}_{2}(1-z)+\ln z \mathrm{Li}_{2}(1-z)-\ln z \mathrm{Li}_{2}(-z)+2 \mathrm{Li}_{3}(1-z)+\mathrm{S}_{12}(1-z)+\mathrm{S}_{12}\left(z^{2}\right)\right)\right\}\right)$. 
Here the factor $(-1)^{n}$ originates from the non-planar diagrams (namely $k$ and $o$ in Fig. 2 in the singlet paper of [6]). It multiplies that part of the matrix element which is needed for the mass factorization of physical processes with two identical quarks in the final state.

The unphysical part is given by [see Eq. (2.14)]

$$
\begin{aligned}
& \hat{A}_{q q}^{\mathrm{NS}, \mathrm{EOM}}\left(z, \frac{-p^{2}}{\mu^{2}}, \frac{1}{\varepsilon}\right) \\
& =F C_{F}\{4 z-2(1-\hat{\xi})+\varepsilon[2 z-(1-\hat{\xi})][1+\ln z+\ln (1-z)]\}+F^{2}\left\{\frac { 1 } { \varepsilon } \left[C_{F}^{2}\{-16-8 z+16 z[-2 \ln (1-z)+\ln z]\}\right.\right. \\
& \left.+C_{A} C_{F}\left(\frac{20}{3}-\frac{88}{3} z\right)+n_{f} C_{F} T_{f}\left(-\frac{16}{3}+\frac{32}{3} z\right)\right]+C_{F}^{2}\left[-\frac{32}{3}-\frac{16}{3} z+\zeta(2)\left(-8+16 z-\frac{32}{3} z^{2}\right)-44 z \ln (1-z)\right. \\
& -24 z \ln ^{2}(1-z)-\frac{4}{3} \ln z(10-17 z)+8(1-4 z) \ln z \ln (1-z)+\frac{16}{3}\left(\frac{1}{z}-3 z-2 z^{2}\right)\left[\ln z \ln (1+z)+\operatorname{Li}_{2}(-z)\right] \\
& +\ln ^{2} z\left(12 z+\frac{16}{3} z^{2}\right)+16 \operatorname{Li}_{2}(1-z)(1-3 z)+8 z(1-z)\left\{-2 \ln (1-z) \zeta(2)+2 \ln z \zeta(2)-\ln ^{2} z \ln (1-z)\right. \\
& \left.\left.-4 \ln z \mathrm{Li}_{2}(1-z)-2 \ln (1-z) \mathrm{Li}_{2}(1-z)+6 \zeta(3)+6 \mathrm{Li}_{3}(1-z)-6 \mathrm{~S}_{12}(1-z)\right\}\right]+C_{A} C_{F}\left[-\frac{26}{9}+\frac{232}{9} z\right. \\
& +4 \zeta(2)\left(1-2 z+\frac{4}{3} z^{2}\right)+\ln z(6-28 z)-\frac{16}{3} \ln (1-z)(1+4 z)-4\left[\ln z \ln (1-z)+2 \operatorname{Li}_{2}(1-z)\right](1-2 z)-\frac{8}{3} z^{2} \ln { }^{2} z \\
& -\frac{8}{3}\left(\frac{1}{z}-3 z-2 z^{2}\right)\left[\ln z \ln (1+z)+\operatorname{Li}_{2}(-z)\right]+8 z(1-z)\left(\frac{1}{2} \ln ^{2} z \ln (1-z)+\zeta(2)[\ln (1-z)-\ln z]\right. \\
& \left.\left.+2 \ln z \mathrm{Li}_{2}(1-z)-3 \zeta(3)+\ln (1-z) \mathrm{Li}_{2}(1-z)-3 \mathrm{Li}_{3}(1-z)+3 \mathrm{~S}_{12}(1-z)\right)\right]+n_{f} C_{F} T_{f}\left[-\frac{8}{9}(1+4 z)\right. \\
& \left.-\frac{8}{3}(1-2 z)\{\ln z+2 \ln (1-z)\}\right]-\frac{16}{3}(-1)^{n}\left(C_{F}^{2}-\frac{1}{2} C_{A} C_{F}\right)\left[1-z+z^{2}\left[-2 \zeta(2)+\ln ^{2} z\right]-\ln z(1-2 z)\right. \\
& \left.\left.+\left(\frac{1}{z}-3 z-2 z^{2}\right)\left[\ln z \ln (1+z)+\operatorname{Li}_{2}(-z)\right]\right]\right\}
\end{aligned}
$$

The purely singlet (PS) OME is split in the same way as the non-singlet one in Eq. (2.7). For the physical part we have [see Eq. (2.16)]

$$
\begin{aligned}
\hat{A}_{q q}^{\mathrm{PS}, \mathrm{PHYS}}\left(z, \frac{-p^{2}}{\mu^{2}}, \frac{1}{\varepsilon}\right)= & F^{2} n_{f} C_{F} T_{f}\left\{\frac{16}{\varepsilon^{2}}\left(1+\frac{4}{3 z}-z-\frac{4}{3} z^{2}+2 \ln z(1+z)\right)+\frac{8}{\varepsilon}\left[\frac{2}{3}\left(16+\frac{5}{z}-13 z-8 z^{2}\right)+2 \ln (1-z)\left(1+\frac{4}{3 z}\right.\right.\right. \\
& \left.-z-\frac{4}{3} z^{2}\right)+\ln z\left(13+\frac{8}{3 z}+13 z\right)+4(1+z)\left(\ln z \ln (1-z)+\frac{3}{4} \ln ^{2} z+\mathrm{Li}_{2}(1-z)\right)+\frac{316}{3}+\frac{20}{z} \\
& -\frac{256}{3} z-40 z^{2}+4\{\zeta(2)+2 \ln (1-z)\}\left(1+\frac{4}{3 z}-z-\frac{4}{3} z^{2}\right)+\frac{16}{3} \ln (1-z)\left(16+\frac{5}{z}-13 z-8 z^{2}\right) \\
& +2 \ln ^{2} z\left(37+\frac{16}{3 z}+41 z+\frac{8}{3} z^{2}\right)+8 \ln z \ln (1-z)\left(13+\frac{8}{3 z}+13 z\right)+\frac{8}{3} \ln z\left(67+\frac{10}{z}+50 z\right) \\
& +16 \operatorname{Li}_{2}(1-z)\left(6+\frac{2}{3 z}+7 z+\frac{2}{3} z^{2}\right)+16(1+z)\left(\frac{1}{2} \ln z \zeta(2)+2 \ln (1-z) \mathrm{Li}_{2}(1-z)\right. \\
& \left.\left.+\ln z \ln ^{2}(1-z)+\frac{3}{2} \ln ^{2} z \ln (1-z)+\frac{7}{12} \ln ^{3} z+2 \ln z \mathrm{Li}_{2}(1-z)-2 \mathrm{Li}_{3}(1-z)+\mathrm{S}_{12}(1-z)\right)\right\},(\mathrm{A} 5)
\end{aligned}
$$

and the unphysical part [see Eq. (2.17)] becomes equal to 


$$
\begin{aligned}
\hat{\mathrm{A}}_{\mathrm{qq}}^{\mathrm{PS}, \mathrm{EOM}}\left(z, \frac{-p^{2}}{\mu^{2}}, \frac{1}{\varepsilon}\right)= & F^{2} n_{f} C_{F} T_{f}\left[\frac{32}{\varepsilon}\left\{-2+z+z^{2}-\ln z(1+2 z)\right\}-72+32 z+40 z^{2}+32 \ln (1-z)\left(-2+z+z^{2}\right)\right. \\
& \left.-16 \ln z(6+7 z)-32(1+2 z)\left(\ln z \ln (1-z)+\frac{3}{4} \ln ^{2} z+\mathrm{Li}_{2}(1-z)\right)\right] .
\end{aligned}
$$

The next OME is split into three pieces according to Eq. (2.18). The physical part is given by [see Eq. (2.27)]

$$
\begin{aligned}
& \hat{A}_{q g}^{\mathrm{PHYS}}\left(z, \frac{-p^{2}}{\mu^{2}}, \frac{1}{\varepsilon}\right)=F n_{f} T_{f}\left[\frac{8}{\varepsilon}\left\{1-2 z+2 z^{2}\right\}+4+4\left(1-2 z+2 z^{2}\right)[\ln z+\ln (1-z)]+\varepsilon\left(2[\ln z+\ln (1-z)]+\left(1-2 z+2 z^{2}\right)\right.\right. \\
& \left.\left.\times\left[\zeta(2)+\{\ln z+\ln (1-z)\}^{2}\right]\right)\right]+F^{2} n_{f}\left(\frac { 1 } { \varepsilon ^ { 2 } } \left\{C _ { A } T _ { f } \left[\frac{16}{3}\left(14+\frac{4}{z}+2 z-9 z^{2}\right)+32 \ln z(1+4 z)\right.\right.\right. \\
& \left.+32 \ln (1-z)\left(1-2 z+2 z^{2}\right)\right]-n_{f} T_{f}^{2} \frac{64}{3}\left[1-2 z+2 z^{2}\right]-8 C_{F} T_{f}\left[1-4 z-4 \ln (1-z)\left(1-2 z+2 z^{2}\right)\right. \\
& \left.\left.+2 \ln z\left(1-2 z+4 z^{2}\right)\right]\right\}+\frac{1}{\varepsilon}\left\{C _ { A } T _ { f } \left[\frac{8}{9}\left(26+\frac{30}{z}-79 z-20 z^{2}\right)+24 \ln ^{2}(1-z)\left(1-2 z+2 z^{2}\right)\right.\right. \\
& +32 \zeta(2)\left(1-z+2 z^{2}\right)+\frac{8}{3} \ln (1-z)\left(17+\frac{8}{z}+38 z-52 z^{2}\right)+32(1+4 z) \ln z \ln (1-z) \\
& +8 \ln ^{2} z(3+10 z)+\frac{16}{3} \ln z\left(25+\frac{4}{z}+13 z+14 z^{2}\right)+64 z(3-z) \operatorname{Li}_{2}(1-z)+16\left(1+2 z+2 z^{2}\right) \\
& \left.\times\left[\ln z \ln (1+z)+\mathrm{Li}_{2}(-z)\right]\right]+C_{F} T_{f}\left[-32+36 z+8\left(1-2 z+2 z^{2}\right)\left[-2 \zeta(2)+3 \ln ^{2}(1-z)\right]\right. \\
& -4 \ln z\left(5+4 z+8 z^{2}\right)+8 \ln (1-z)\left(3+4 z^{2}\right)-12 \ln ^{2} z\left(1-2 z+4 z^{2}\right)+32 \ln z \ln (1-z)\left(1-2 z+z^{2}\right) \\
& \left.\left.+16 \mathrm{Li}_{2}(1-z)\left(3-6 z+4 z^{2}\right)\right]+n_{f} T_{f}^{2} \frac{32}{3}\left[\frac{1}{3}\left(2-10 z+10 z^{2}\right)-\left(1-2 z+2 z^{2}\right)\{\ln z+\ln (1-z)\}\right]\right\} \\
& +C_{A} T_{f}\left[\frac{2900}{27}+\frac{20}{z}-\frac{136}{27} z-\frac{2492}{27} z^{2}+\zeta(2)\left(12+\frac{16}{3 z}+16 z-\frac{172}{3} z^{2}+16 z^{3}\right)+8 \zeta(3)\left(1-z-4 z^{2}+6 z^{3}\right)\right. \\
& +\frac{28}{3} \ln ^{3}(1-z)\left(1-2 z+2 z^{2}\right)+\frac{2}{3} \ln ^{2}(1-z)\left(23+\frac{16}{z}+110 z-138 z^{2}\right)+8 \ln (1-z) \operatorname{Li}_{2}(1-z) \\
& \times\left(4+23 z+2 z^{2}-2 z^{3}\right)+\frac{4}{9} \ln (1-z)\left(68+\frac{60}{z}-283 z+94 z^{2}\right)+8 \ln (1-z) \zeta(2)\left(3-7 z+8 z^{2}-2 z^{3}\right) \\
& +8 \ln z \ln ^{2}(1-z)\left(3+10 z+2 z^{2}\right)+\frac{4}{3} \ln z \ln (1-z)\left(83+\frac{16}{z}+86 z+22 z^{2}\right)+4 \ln ^{2} z \ln (1-z)(5+21 z \\
& \left.-2 z^{3}\right)+\frac{4}{3} \ln ^{3} z(7+22 z)+\frac{4}{3} \ln ^{2} z\left(61+\frac{8}{z}+49 z+51 z^{2}-6 z^{3}\right)+16 \ln z \operatorname{Li}_{2}(1-z)\left(1+7 z-2 z^{3}\right) \\
& +\frac{8}{9} \ln z\left(157+\frac{30}{z}+127 z+59 z^{2}\right)+8 \ln z \zeta(2)\left(2+3 z+2 z^{3}\right) \\
& +16 \mathrm{Li}_{2}(1-z)\left(5+\frac{2}{3 z}+4 z+\frac{37}{6} z^{2}\right)-8\left(1+4 z+z^{2}-2 z^{3}\right)\left[\ln z \ln (1+z)+\mathrm{Li}_{2}(-z)\right]-8 \mathrm{Li}_{3}(1-z) \\
& \times\left(8+13 z+14 z^{2}-6 z^{3}\right)+8 \mathrm{~S}_{12}(1-z)\left(5-5 z+12 z^{2}-6 z^{3}\right)+8\left(1+2 z+2 z^{2}\right)\left\{2 \ln (1+z) \operatorname{Li}_{2}(1-z)\right. \\
& +4 \ln (1+z) \operatorname{Li}_{2}(-z)+2 \ln (1+z) \zeta(2)+2 \ln z \ln (1-z) \ln (1+z)+2 \ln z \ln ^{2}(1+z)+\frac{1}{2} \ln ^{2} z \ln (1+z) \\
& \left.\left.+\ln z \mathrm{Li}_{2}(-z)-\mathrm{Li}_{3}(-z)+6 \mathrm{~S}_{12}(-z)-\mathrm{S}_{12}\left(z^{2}\right)\right\}\right]+C_{F} T_{f}\left[-34+58 z-2 \zeta(2)\left(9-12 z+8 z^{2}\right)\right.
\end{aligned}
$$




$$
\begin{aligned}
& +4\left(1-2 z+2 z^{2}\right)\left[-10 \zeta(3)+\frac{7}{3} \ln ^{3}(1-z)-2 \ln (1-z) \zeta(2)\right]+4 \ln ^{2}(1-z)\left(5-2 z+6 z^{2}\right) \\
& +8 \ln (1-z) \mathrm{Li}_{2}(1-z)\left(5-10 z+6 z^{2}\right)-16 z(1-3 z) \ln (1-z)+4 \ln z \ln ^{2}(1-z)\left(7-14 z+10 z^{2}\right) \\
& +4 \ln z \ln (1-z)\left(7-16 z+4 z^{2}\right)+4 \ln ^{2} z \ln (1-z)\left(5-10 z+4 z^{2}\right)-\frac{14}{3} \ln ^{3} z\left(1-2 z+4 z^{2}\right) \\
& -\ln ^{2} z\left(13+20 z+24 z^{2}\right)+32 \ln z \operatorname{Li}_{2}(1-z)\left(2-4 z+3 z^{2}\right)-2 \ln z\left(34-15 z+24 z^{2}\right)-4 \ln z \zeta(2) \\
& \left.\times\left(5-10 z+12 z^{2}\right)+8\left(3-6 z+2 z^{2}\right)\left[2 \operatorname{Li}_{2}(1-z)-\operatorname{Li}_{3}(1-z)\right]+16 \mathrm{~S}_{12}(1-z)\left(3-6 z+5 z^{2}\right)\right] \\
& \left.-n_{f} T_{f}^{2} \frac{8}{3}\left[\frac{2}{9}\left(13-56 z+56 z^{2}\right)-\frac{4}{3}\left(1-5 z+5 z^{2}\right)[\ln z+\ln (1-z)]+(1-2 z+2 z)\{\ln z+\ln (1-z)\}^{2}\right]\right) .
\end{aligned}
$$

The unphysical part (EOM) [see Eq. (2.28)] becomes equal to

$$
\begin{aligned}
& \hat{A}_{q g}^{\mathrm{EOM}}\left(z, \frac{-p^{2}}{\mu^{2}}, \frac{1}{\varepsilon}\right)=F n_{f} T_{f}\{-16 z(1-z)-8 \varepsilon z(1-z)[\ln z+\ln (1-z)]\}+F^{2} n_{f}\left\{\frac { 1 } { \varepsilon } \left[C _ { A } T _ { f } \left(48+\frac{128}{3} z-\frac{272}{3} z^{2}\right.\right.\right. \\
& \left.+16 \ln z\left(1+8 z+8 z^{2}\right)\right)+C_{F} T_{f}[-32+32 z-128 z(1-z) \ln (1-z)+64 z(1-2 z) \ln z] \\
& \left.+n_{f} T_{f}^{2} \frac{128}{3} z(1-z)\right]+C_{A} T_{f}\left[68-\frac{296}{9} z-\frac{316}{9} z^{2}+32 \zeta(2)\left(-z+z^{2}+z^{3}\right)\right. \\
& +16 \zeta(3)\left(z-11 z^{2}+6 z^{3}\right)+16 \ln (1-z)\left(3+\frac{25}{3} z-\frac{34}{3} z^{2}\right)+16 \ln (1-z) \zeta(2)\left(z+z^{2}-2 z^{3}\right) \\
& +16 \ln z \ln (1-z)\left(1+10 z+6 z^{2}\right)+4 \ln ^{2} z\left(3+24 z+24 z^{2}-4 z^{3}\right)-32 z(1-z) \ln z \operatorname{Li}_{2}(-z) \\
& +8 \ln z\left(7+\frac{50}{3} z+\frac{34}{3} z^{2}\right)+16 \mathrm{Li}_{2}(1-z)\left(1+12 z+4 z^{2}\right)+32\left(-1+2 z^{2}+z^{3}\right)\left[\ln z \ln (1+z)+\mathrm{Li}_{2}(-z)\right] \\
& +16\left(z-3 z^{2}+2 z^{3}\right)\left(-\ln (1-z) \operatorname{Li}_{2}(1-z)-\frac{1}{2} \ln ^{2} z \ln (1-z)-2 \ln z \operatorname{Li}_{2}(1-z)\right. \\
& \left.+\ln z \zeta(2)+3 \operatorname{Li}_{3}(1-z)\right)-16 \mathrm{~S}_{12}(1-z)\left(5 z-11 z^{2}+6 z^{3}\right)+32 z(1-3 z) \operatorname{Li}_{3}(-z) \\
& \left.+32 z(1+z)\left(-\frac{1}{2} \ln ^{2} z \ln (1+z)+\ln z \ln ^{2}(1+z)+\ln (1+z) \zeta(2)+2 \ln (1+z) \operatorname{Li}_{2}(-z)+2 \mathrm{~S}_{12}(-z)\right)\right] \\
& +C_{F} T_{f}\left[-16(1-z)+32 z(1-z)\left[2 \zeta(2)-3 \ln ^{2}(1-z)\right]-32 \ln (1-z)\left(1+3 z-4 z^{2}\right)\right. \\
& -64 z(2-z) \ln z \ln (1-z)+48 z(1-2 z) \ln ^{2} z-16 \ln z\left(1-z+8 z^{2}\right) \\
& \left.\left.-64 z(3-2 z) \operatorname{Li}_{2}(1-z)\right]+n_{f} T_{f}^{2} \frac{64}{3} z(1-z)\left(-\frac{5}{3}+\ln z+\ln (1-z)\right)\right\}
\end{aligned}
$$

The part which is due to the contribution of the non-gauge invariant (NGI) operator $O_{A}$ [see Eq. (2.29)] is equal to 


$$
\begin{aligned}
\hat{A}_{q g}^{\mathrm{NGI}}\left(z, \frac{-p^{2}}{\mu^{2}}, \frac{1}{\varepsilon}\right)= & F^{2} n_{f} C_{A} T_{f}\left[-\frac{16}{\varepsilon^{2}}\left\{\frac{2}{3 z}-z+\frac{1}{3} z^{2}+\ln z\right\}+\frac{1}{\varepsilon}\left\{-\frac{8}{3}\left(7+\frac{5}{z}-13 z+z^{2}\right)-\frac{16}{3} \ln (1-z)\left(\frac{2}{z}-3 z+z^{2}\right)\right.\right. \\
& \left.-16 \ln z \ln (1-z)-12 \ln ^{2} z-8 \ln z\left(5+\frac{4}{3 z}+z\right)-16 \operatorname{Li}_{2}(1-z)\right\}-\frac{104}{3}-\frac{10}{z}+\frac{122}{3} z+4 z^{2} \\
& -\frac{4}{3}\left(\frac{2}{z}-3 z+z^{2}\right)\left[\zeta(2)+2 \ln ^{2}(1-z)\right]-16 \ln (1-z) \operatorname{Li}_{2}(1-z)-\frac{8}{3} \ln (1-z)\left(7+\frac{5}{z}-13 z+z^{2}\right) \\
& -8 \ln z \ln ^{2}(1-z)-8 \ln z(1-z)\left(5+\frac{4}{3 z}+z\right)-12 \ln ^{2} z \ln (1-z)-\frac{14}{3} \ln ^{3} z \\
& -2 \ln ^{2} z\left(15+\frac{8}{3 z}+5 z-\frac{2}{3} z^{2}\right)-16 \ln z \operatorname{Li}_{2}(1-z)-\frac{8}{3} \ln z\left(23+\frac{5}{z}+10 z\right) \\
& \left.-4 \operatorname{Li}_{2}(1-z)\left(10+\frac{4}{3 z}+4 z-\frac{2}{3} z^{2}\right)-4 \ln z \zeta(2)+16 \operatorname{Li}_{3}(1-z)-8 \mathrm{~S}_{12}(1-z)\right]
\end{aligned}
$$

The next OME is split into physical and unphysical parts according to Eq. (2.30). The former [see Eq. (2.31)] is

$$
\begin{aligned}
& \hat{A}_{g q}^{\mathrm{PHYS}}\left(z, \frac{-p^{2}}{\mu^{2}}, \frac{1}{\varepsilon}\right)=F C_{F}\left\{\frac{4}{\varepsilon}\left(z-2+\frac{2}{z}\right)-6+\frac{4}{z}+6 z-\left(4-\frac{4}{z}-2 z\right)[\ln z+\ln (1-z)]+\varepsilon\left[-1+2 z+\left(-3+\frac{2}{z}+3 z\right)\right.\right. \\
& \left.\left.\times[\ln z+\ln (1-z)]+\left(-1+\frac{1}{z}+\frac{z}{2}\right)\left[\zeta(2)+\{\ln z+\ln (1-z)\}^{2}\right]\right]\right\}+F^{2}\left(\frac { 1 } { \varepsilon ^ { 2 } } \left\{C_{F}^{2}[16-4 z-16 \ln (1-z)\right.\right. \\
& \left.\times\left(2-\frac{2}{z}-z\right)+8 \ln z(2-z)\right]+C_{A} C_{F}\left[\frac{16}{3}-\frac{24}{z}+\frac{112}{3} z+\frac{32}{3} z^{2}+16 \ln (1-z)\left(-2+\frac{2}{z}+z\right)-32 \ln z\right. \\
& \left.\left.\times\left(1+\frac{1}{z}+z\right)\right]-n_{f} C_{F} T_{f} \frac{32}{3}\left(-2+\frac{2}{z}+z\right)\right\}+\frac{1}{\varepsilon}\left\{C _ { F } ^ { 2 } \left[34-38 z+4\left(-2+\frac{2}{z}+z\right)\right.\right. \\
& \times\left\{4 \zeta(2)+3 \ln ^{2}(1-z)\right\}+4 \ln (1-z)(4+z)+2(2-z)\left\{4 \ln z \ln (1-z)+3 \ln ^{2} z\right\}+2 \ln z(24+5 z) \\
& \left.-8 \mathrm{Li}_{2}(1-z)\left(-6+\frac{4}{z}+3 z\right)\right]+C_{A} C_{F}\left[-\frac{604}{9}-\frac{212}{9 z}+\frac{668}{9} z+\frac{80}{3} z^{2}-16 \zeta(2)\left(-1+\frac{2}{z}+z\right)\right. \\
& +12 \ln ^{2}(1-z)\left(-2+\frac{2}{z}+z\right)-\frac{4}{3} \ln (1-z)\left(10+\frac{16}{z}-47 z-8 z^{2}\right)-8 \ln z \ln (1-z)\left(10-\frac{2}{z}+z\right) \\
& -\ln z\left(\frac{296}{3}+\frac{24}{z}+\frac{212}{3} z\right)-4 \ln ^{2} z\left(6+\frac{4}{z}+5 z\right)-32 \operatorname{Li}_{2}(1-z)\left(3-\frac{1}{z}\right)-8\left(2+\frac{2}{z}+z\right)\{\ln z \ln (1+z) \\
& \left.\left.\left.+\mathrm{Li}_{2}(-z)\right\}\right]+n_{f} C_{F} T_{f}\left[\frac{32}{9}\left(4-\frac{1}{z}-5 z\right)+\frac{16}{3}\left(2-\frac{2}{z}-z\right)\{\ln (1-z)+2 \ln z\}\right]\right\}+C_{F}^{2}[38-29 z+\zeta(2) \\
& \times\left(-16+\frac{4}{z}+17 z\right)+16 \zeta(3)\left(1-\frac{1}{z}+z\right)+4 \ln (1-z) \zeta(2)\left(-10+\frac{10}{z}+3 z\right)+\frac{14}{3} \ln ^{3}(1-z) \\
& \times\left(-2+\frac{2}{z}+z\right)+4 \ln ^{2}(1-z)(2+z)-8 \ln (1-z) \operatorname{Li}_{2}(1-z)\left(-4+\frac{2}{z}+3 z\right)+4 \ln (1-z)\left(13-\frac{2}{z}-11 z\right) \\
& +4 \ln z \ln (1-z)\left(17-\frac{1}{z}\right)+2 \ln ^{2} z \ln (1-z)(6-5 z)+(2-z)\left(4 \ln z \ln ^{2}(1-z)+\frac{7}{3} \ln ^{3} z\right) \\
& +\ln ^{2} z\left(32+\frac{17}{2} z\right)+8 \ln z \operatorname{Li}_{2}(1-z)(2-3 z)+33(1+z) \ln z+2 \ln z \zeta(2)(2+3 z) \\
& \left.-8 \operatorname{Li}_{2}(1-z)\left(-10+\frac{1}{z}+2 z\right)-8 \operatorname{Li}_{3}(1-z)\left(\frac{2}{z}-3 z\right)-4 \mathrm{~S}_{12}(1-z)\left(6-\frac{8}{z}+3 z\right)\right]
\end{aligned}
$$




$$
\begin{aligned}
& +C_{A} C_{F}\left[\frac{1}{27}\left(319-\frac{2539}{z}+1678 z+828 z^{2}\right)-4 \zeta(3)\left(8-\frac{1}{z}-11 z+6 z^{2}\right)+\frac{2}{3} \zeta(2)\left(20-\frac{15}{z}-16 z+4 z^{2}\right)\right. \\
& +4 \ln (1-z) \zeta(2)\left(10-\frac{7}{z}-7 z+2 z^{2}\right)-\frac{14}{3} \ln ^{3}(1-z)\left(2-\frac{2}{z}-z\right)-\frac{1}{3} \ln ^{2}(1-z)\left(34+\frac{30}{z}-113 z-16 z^{2}\right) \\
& -4 \ln (1-z) \operatorname{Li}_{2}(1-z)\left(22-\frac{1}{z}+7 z-2 z^{2}\right)-\frac{2}{9} \ln (1-z)\left(454-\frac{22}{z}-425 z-120 z^{2}\right) \\
& -4 \ln z \zeta(2)\left(4+\frac{3}{z}-z+2 z^{2}\right)-2 \ln z \ln ^{2}(1-z)\left(30-\frac{6}{z}+z\right)-\frac{4}{3} \ln z \ln (1-z)\left(97+\frac{10}{z}+25 z\right) \\
& -2 \ln ^{2} z \ln (1-z)\left(22-\frac{5}{z}+7 z-2 z^{2}\right)-\frac{2}{3} \ln ^{3} z\left(14+\frac{8}{z}+11 z\right)-\frac{1}{3} \ln ^{2} z\left(226+\frac{42}{z}+175 z+8 z^{2}\right) \\
& -8 \ln z \operatorname{Li}_{2}(1-z)\left(8-\frac{5}{z}+3 z-2 z^{2}\right)-\frac{1}{9} \ln z\left(1546+\frac{680}{z}+856 z\right)-4 \operatorname{Li}_{2}(1-z)\left(36-\frac{5}{3 z}+10 z\right. \\
& \left.+\frac{4}{3} z^{2}\right)+4\left(1-\frac{1}{z^{2}}+\frac{1}{z}-2 z\right)\left\{\ln z \ln (1+z)+\operatorname{Li}_{2}(-z)\right\}+4 \operatorname{Li}_{3}(1-z)\left(10+\frac{5}{z}+17 z-6 z^{2}\right) \\
& -4 \mathrm{~S}_{12}(1-z)\left(4-\frac{9}{z}+8 z-6 z^{2}\right)+4\left(2+\frac{2}{z}+z\right)\left\{-2 \ln (1+z) \zeta(2)-2 \ln (1+z) \mathrm{Li}_{2}(1-z)\right. \\
& -4 \ln (1+z) \mathrm{Li}_{2}(-z)-2 \ln z \ln (1-z) \ln (1+z)-2 \ln z \ln ^{2}(1+z)-\frac{1}{2} \ln ^{2} z \ln (1+z) \\
& \left.\left.-\ln z \mathrm{Li}_{2}(-z)+\mathrm{Li}_{3}(-z)-6 \mathrm{~S}_{12}(-z)+\mathrm{S}_{12}\left(z^{2}\right)\right\}\right]+n_{f} C_{F} T_{f}\left[\frac{8}{27}\left(38-\frac{32}{z}-25 z\right)\right. \\
& \left.\left.+\frac{4}{3}\left(2-\frac{2}{z}-z\right)\left[\{2 \ln z+\ln (1-z)\}^{2}+2 \zeta(2)\right]+\frac{16}{9}\left(4-\frac{1}{z}-5 z\right)\{\ln (1-z)+2 \ln z\}\right]\right) .
\end{aligned}
$$

The unphysical part is given by [see Eq. (2.32)]

$$
\begin{aligned}
\hat{A}_{g q}^{\mathrm{EOM}}\left(z, \frac{-p^{2}}{\mu^{2}}, \frac{1}{\varepsilon}\right)= & F C_{F}\{4(1-z)+2 \varepsilon(1-z)[1+\ln z+\ln (1-z)]\}+F^{2}\left\{\frac { 1 } { \varepsilon } \left[16 C_{F}^{2}\left(-2+\frac{1}{z}+z-z \ln z\right)-n_{f} C_{F} T_{f} \frac{64}{3}\right.\right. \\
& \left.\times(1-z)+16 C_{A} C_{F}\left(\frac{14}{3}(1-z)+\frac{1}{z}-z^{2}+2(1-z) \ln (1-z)+2(1+2 z) \ln z\right)\right]+C_{F}^{2}\left(-8\left(1-\frac{1}{z}\right)\right. \\
& +16(1-z) \zeta(2)-4 \ln (1-z)\left(9-\frac{4}{z}-5 z\right)-12 z \ln ^{2} z-16 \ln z \ln (1-z)-8 \ln z\left(2-\frac{1}{z}+2 z\right) \\
& \left.-16(2-z) \mathrm{Li}_{2}(1-z)\right]+C_{A} C_{F}\left[\frac{400}{9}-\frac{28}{3 z}-\frac{64}{9} z-28 z^{2}+24(1-z) \ln { }^{2}(1-z)+16 \ln (1-z)\left(\frac{16}{3}+\frac{1}{z}\right.\right. \\
& \left.-\frac{16}{3} z-z^{2}\right)+24(3+z) \ln z \ln (1-z)+8 \ln z\left(3+\frac{16}{3} z\right)+2 \ln z\left(55+\frac{2}{3 z}+\frac{80}{3} z\right)+16(5+z) \mathrm{Li}_{2}(1-z) \\
& -8 \zeta(2)\left(1-\frac{7}{3} z\right)+4\left(3-\frac{1}{3 z^{2}}+\frac{8}{3} z\right)\left\{\ln z \ln (1+z)+\mathrm{Li}_{2}(-z)\right\}-4\left(1-3 z+2 z^{2}\right)(-\ln z \zeta(2) \\
& +\ln (1-z) \mathrm{Li}_{2}(1-z)+\frac{1}{2} \ln z \ln (1-z)+\ln (1-z) \zeta(2)-3 \zeta(3)+2 \ln z \operatorname{Li}_{2}(1-z)-3 \mathrm{Li}_{3}(1-z) \\
& \left.\left.\left.+3 S_{12}(1-z)\right)\right]-n_{f} C_{F} T_{f} \frac{32}{3}(1-z)\left(\frac{1}{3}+\ln (1-z)+2 \ln z\right)\right\}
\end{aligned}
$$


The gluonic OME can be split into three parts according to Eq. (2.33). The physical part is equal to [see Eq. (2.34)]

$$
\begin{aligned}
& \hat{A}_{g g}^{\mathrm{PHYS}}\left(z, \frac{-p^{2}}{\mu^{2}}, \frac{1}{\varepsilon}\right)=\delta(1-z)+F\left(\frac{1}{\varepsilon}\left[C_{A}\left(-16+\frac{8}{z}+8 z-8 z^{2}+\frac{8}{1-z}+\frac{22}{3} \delta(1-z)\right)-\frac{8}{3} n_{f} T_{f} \delta(1-z)\right]+C_{A}\left[-6+\frac{4}{z}+4 z\right.\right. \\
& -4 z^{2}-4\left(2-\frac{1}{z}-z+z^{2}+\frac{4}{1-z}\right)[\ln z+\ln (1-z)]+\frac{1-\hat{\xi}}{1-z}+\delta(1-z)\left(-\frac{67}{9}+4 \zeta(2)-(1-\hat{\xi})\right. \\
& \left.\left.+\frac{1}{4}(1-\hat{\xi})^{2}\right)\right]+n_{f} T_{f} \frac{20}{9} \delta(1-z)+\varepsilon\left\{C _ { A } \left[\left(-2+\frac{1}{z}+z-z^{2}+\frac{1}{1-z}\right)\left\{\zeta(2)+[\ln z+\ln (1-z)]^{2}\right\}\right.\right. \\
& -\left(3-\frac{2}{z}-2 z+2 z^{2}\right)[\ln (1-z)+\ln z]-\frac{1}{2} \frac{1-\hat{\xi}}{1-z}\{1-\ln z-\ln (1-z)\}+\delta(1-z)\left(\frac{202}{27}-\frac{11}{12} \zeta(2)\right. \\
& \left.\left.\left.\left.-\frac{14}{3} \zeta(3)+\frac{1}{4} \zeta(2)(1-\hat{\xi})-\frac{1}{4}(1-\hat{\xi})^{2}\right)\right]-n_{f} T_{f} \delta(1-z)\left(\frac{56}{27}-\frac{1}{3} \zeta(2)\right)\right\}\right)+F^{2}\left(\frac { 1 } { \varepsilon ^ { 2 } } \left\{C_{A}^{2}[-8(10+z)\right.\right. \\
& -\frac{88}{3}\left(\frac{1}{z}-z^{2}\right)+\frac{88}{1-z}+\delta(1-z)\left(\frac{484}{9}-32 \zeta(2)\right)-64 \ln (1-z)\left(2-\frac{1}{z}-z+z^{2}-\frac{1}{1-z}\right) \\
& \left.-32 \ln z\left(\frac{1}{z}+3 z-z^{2}+\frac{1}{1-z}\right)\right]+n_{f} C_{A} T_{f}\left[32\left(2-\frac{1}{z}-z+z^{2}-\frac{1}{1-z}\right)-\frac{352}{9} \delta(1-z)\right] \\
& \left.+16 n_{f} C_{F} T_{f}\left[1-z+\frac{4}{3}\left(\frac{1}{z}-z^{2}\right)+2 \ln z(1+z)\right]+n_{f}^{2} T_{f}^{2} \frac{64}{9} \delta(1-z)\right\}+\frac{1}{\varepsilon}\left\{C _ { A } ^ { 2 } \left[\frac{2}{3}\left(53-\frac{46}{z}+55 z+28 z^{2}\right)\right.\right. \\
& -\frac{86}{1-z}-8 \zeta(2)\left(4+2 z^{2}-\frac{1}{1+z}-\frac{1}{1-z}\right)+\delta(1-z)\left(-\frac{3326}{27}+\frac{176}{3} \zeta(2)+20 \zeta(3)\right)-48 \ln ^{2}(1-z) \\
& \times\left(2-\frac{1}{z}-z+z^{2}-\frac{1}{1-z}\right)-\frac{16}{3} \ln (1-z)\left(13+\frac{5}{z}+z-5 z^{2}-\frac{11}{1-z}\right)-16 \ln z \ln (1-z)\left(6-\frac{1}{z}+3 z\right. \\
& \left.+z^{2}-\frac{1}{1-z}\right)-4 \ln ^{2} z\left(\frac{4}{z}+16 z-6 z^{2}+\frac{1}{1+z}+\frac{5}{1-z}\right)-4 \ln z\left(36+\frac{6}{z}+21 z-\frac{44}{3} \frac{1}{1-z}\right)-64(1+z) \\
& \left.\times \mathrm{Li}_{2}(1-z)-16\left(2+\frac{1}{z}+z+z^{2}-\frac{1}{1+z}\right)\left\{\ln z \ln (1+z)+\mathrm{Li}_{2}(-z)\right\}\right]+n_{f} C_{A} T_{f}\left[-\frac{8}{3}(11-5 z)\right. \\
& +\frac{152}{9}\left(\frac{1}{z}-z^{2}\right)+\frac{24}{1-z}+\frac{64}{3} \ln (1-z)\left(2-\frac{1}{z}-z+z^{2}-\frac{1}{1-z}\right)+\frac{16}{3} \ln z\left(9-\frac{4}{z}-3 z+4 z^{2}-\frac{4}{1-z}\right) \\
& \left.+\delta(1-z)\left(\frac{2168}{27}-\frac{64}{3} \zeta(2)\right)\right]+n_{f} C_{F} T_{f}\left[\frac{160}{3}(1-z)-\frac{176}{9}\left(\frac{1}{z}-z^{2}\right)+4 \delta(1-z)+16 \ln (1-z)\left(1+\frac{4}{3 z}\right.\right. \\
& \left.\left.-z-\frac{4}{3} z^{2}\right)+8 \ln z\left(5+3 z-\frac{8}{3} z^{2}\right)+8(1+z)\left\{4 \ln z \ln (1-z)+3 \ln ^{2} z+4 \operatorname{Li}_{2}(1-z)\right\}\right]-n_{f}^{2} T_{f}^{2} \frac{320}{27} \\
& \times \delta(1-z)\}+C_{A}^{2}\left[-\frac{1672}{45}-\frac{13951}{135 z}+\frac{187}{45} z+\frac{8686}{135} z^{2}+\frac{254}{3} \frac{1}{1-z}+\zeta(2)\left(\frac{1}{3}-\frac{40}{3 z}-\frac{61}{3} z+26 z^{2}\right.\right. \\
& \left.-\frac{124}{15} z^{3}+\frac{19}{1-z}\right)+\zeta(3)\left(30-\frac{12}{z}-12 z+59 z^{2}-12 z^{3}+\frac{4}{1+z}-\frac{10}{1-z}\right)+\delta(1-z)\left(\frac{11141}{54}-\frac{214}{3} \zeta(2)\right. \\
& \left.+5 \zeta(2)^{2}-\frac{854}{9} \zeta(3)\right)-\frac{56}{3} \ln ^{3}(1-z)\left(2-\frac{1}{z}-z+z^{2}-\frac{1}{1-z}\right)-2 \ln ^{2}(1-z)\left(16+\frac{19}{3 z}+z-\frac{19}{3} z^{2}\right.
\end{aligned}
$$




$$
\begin{aligned}
& \left.-\frac{11}{1-z}\right)-\ln (1-z) \operatorname{Li}_{2}(1-z)\left(102+\frac{12}{z}+76 z+33 z^{2}-4 z^{3}-\frac{16}{1+z}-\frac{2}{1-z}\right)-\frac{1}{9} \ln (1-z)\left(233+\frac{14}{z}\right. \\
& \left.-703 z+148 z^{2}+\frac{506}{1-z}\right)-\ln (1-z) \zeta(2)\left(26-\frac{12}{z} 12 z+25 z^{2}-4 z^{3}-\frac{18}{1-z}\right)-4 \ln z \ln ^{2}(1-z)(22 \\
& \left.-\frac{3}{z}+5 z+7 z^{2}-\frac{2}{1+z}-\frac{5}{1-z}\right)-\frac{1}{3} \ln z \ln (1-z)\left(461+\frac{26}{z}+167 z+64 z^{2}-\frac{97}{1-z}\right) \\
& -4\left(7+\frac{4}{z}+4 z+4 z^{2}-\frac{2}{1+z}\right)\left\{2 \ln (1+z) \operatorname{Li}_{2}(-z)+\ln (1+z) \zeta(2)+\ln z \ln ^{2}(1+z)\right\}-\ln ^{2} z \ln (1-z) \\
& \times\left(51-\frac{10}{z}+38 z+\frac{17}{2} z^{2}-2 z^{3}+\frac{4}{1+z}-\frac{5}{1-z}\right)-2 \ln ^{2} z \ln (1+z)\left(5+\frac{2}{z}+2 z+2 z^{2}-\frac{4}{1+z}\right) \\
& -2 \ln ^{3} z\left(\frac{8}{3 z}+12 z-\frac{14}{3} z^{2}+\frac{1}{1+z}+\frac{11}{3} \frac{1}{1-z}\right)-2 \ln ^{2} z\left(44+\frac{7}{z}+\frac{97}{3} z+\frac{11}{3} z^{2}-\frac{31}{15} z^{3}-\frac{11}{1-z}\right) \\
& -2 \ln z \operatorname{Li}_{2}(1-z)\left(54-\frac{20}{z}+12 z+25 z^{2}-4 z^{3}+\frac{4}{1+z}-\frac{14}{1-z}\right)-4 \ln z \operatorname{Li}_{2}(-z) \\
& \times\left(3+\frac{2}{z}+2 z+2 z^{2}-\frac{2}{1+z}+\frac{2}{1-z}\right)-\ln z\left(\frac{1727}{15}+\frac{3598}{45 z}+\frac{564}{5} z+\frac{848}{45} z^{2}+\frac{521}{9} \frac{1}{1-z}\right) \\
& +\ln z \zeta(2)\left(6-\frac{12}{z}-28 z+25 z^{2}-4 z^{3}-\frac{10}{1-z}\right)-2 \operatorname{Li}_{2}(1-z)\left(67-\frac{28}{3 z}+13 z\right. \\
& \left.+\frac{82}{3} z^{2}+\frac{13}{3} \frac{1}{1-z}\right)+\frac{2}{3}\left(17-\frac{32}{5} \frac{1}{z^{2}}+\frac{10}{z}+11 z-2 z^{2}-\frac{62}{5} z^{3}\right)\left\{\ln z \ln (1+z)+\operatorname{Li}_{2}(-z)\right\}+\operatorname{Li}_{3}(1-z) \\
& \times\left(114+\frac{4}{z}+68 z+67 z^{2}-12 z^{3}-\frac{16}{1+z}-\frac{6}{1-z}\right)+4 \operatorname{Li}_{3}(-z)\left(1+\frac{2}{z}+2 z+2 z^{2}+\frac{4}{1-z}\right)+\mathrm{S}_{12}(1-z) \\
& \times\left(-126+\frac{68}{z}+36 z-91 z^{2}+12 z^{3}-\frac{8}{1+z}+\frac{46}{1-z}\right)-8 S_{12}(-z)\left(11+\frac{6}{z}+6 z+6 z^{2}-\frac{4}{1+z}\right)+8(2+z \\
& \left.\left.+z^{2}-\frac{1}{1+z}+\frac{1}{z}\right)\left\{-2 \ln (1+z) \mathrm{Li}_{2}(1-z)-2 \ln z \ln (1-z) \ln (1+z)+\mathrm{S}_{12}\left(z^{2}\right)\right\}\right]+n_{f} C_{A} T_{f}\left[\frac{4}{3}(43-28 z)\right. \\
& -\frac{356}{9}\left(\frac{1}{z}-z^{2}\right)-\frac{64}{3} \frac{1}{1-z}+\frac{4}{3} \zeta(2)\left(7+\frac{2}{z}+16 z+2 z^{2}-\frac{6}{1-z}\right)+\frac{4}{9} \delta(1-z)\left(-\frac{806}{3}+59 \zeta(2)+34 \zeta(3)\right) \\
& +8 \ln ^{2}(1-z)\left(2-\frac{1}{z}-z+z^{2}-\frac{1}{1-z}\right)-\frac{4}{9} \ln (1-z)\left(41-\frac{30}{z}-7 z+30 z^{2}-\frac{34}{1-z}\right)+\frac{4}{3} \ln z \ln (1-z)(25 \\
& \left.-\frac{12}{z}-14 z+12 z^{2}-\frac{8}{1-z}\right)+4 \ln ^{2} z\left(5-\frac{8}{3 z}-3 z+2 z^{2}-\frac{2}{1-z}\right)-4 \ln z \\
& \times\left(5-\frac{4}{9 z}-2 z+\frac{10}{3} z^{2}-\frac{37}{9} \frac{1}{1-z}\right)+\frac{8}{3} \operatorname{Li}_{2}(1-z)\left(3-\frac{2}{z}-6 z+2 z^{2}+\frac{2}{1-z}\right)+\frac{16}{3}\left(3+\frac{1}{z}+3 z+z^{2}\right) \\
& \times\left\{\ln z \ln (1+z)+\mathrm{Li}_{2}(-z)\right\}+8 z^{2}\left(\ln (1-z) \mathrm{Li}_{2}(1-z)+\ln (1-z) \zeta(2)+\frac{1}{2} \ln ^{2} z \ln (1-z)+2 \ln z \mathrm{Li}_{2}(1\right. \\
& \left.\left.-z)-\ln z \zeta(2)-3 \zeta(3)-3 \operatorname{Li}_{3}(1-z)+3 \mathrm{~S}_{12}(1-z)\right)\right]+n_{f} C_{F} T_{f}\left[-\frac{80}{9}(1-z)\right. \\
& +\frac{1720}{27}\left(\frac{1}{z}-z^{2}\right)+\delta(1-z)\left(-\frac{55}{3}+16 \zeta(3)\right)+4 \zeta(2)\left(1-\frac{4}{3 z}-9 z-\frac{4}{3} z^{2}\right)+8 \ln ^{2}(1-z)
\end{aligned}
$$




$$
\begin{aligned}
& \times\left(1+\frac{4}{3 z}-z-\frac{4}{3} z^{2}\right)+\ln (1-z)\left[\frac{160}{3}(1-z)-\frac{176}{9}\left(\frac{1}{z}-z^{2}\right)\right]+8 \ln z \ln (1-z)\left(5+3 z-\frac{8}{3} z^{2}\right) \\
& +2 \ln ^{2} z\left(13+19 z-\frac{16}{3} z^{2}\right)+8 \ln z\left(\frac{35}{3}+5 z+\frac{22}{9} z^{2}\right)+\frac{32}{3} \mathrm{Li}_{2}(1-z)\left(3-\frac{1}{z}+3 z-z^{2}\right) \\
& -\frac{32}{3}\left(3+\frac{1}{z}+3 z+z^{2}\right)\left\{\ln z \ln (1+z)+\mathrm{Li}_{2}(-z)\right\}+8(1+z)\left(4 \ln (1-z) \mathrm{Li}_{2}(1-z)+2 \ln z \ln ^{2}(1-z)\right. \\
& \left.\left.+3 \ln ^{2} z \ln (1-z)+\frac{7}{6} \ln ^{3} z+4 \ln z \mathrm{Li}_{2}(1-z)+\ln z \zeta(2)-4 \mathrm{Li}_{3}(1-z)+2 \mathrm{~S}_{12}(1-z)\right)\right] \\
& \left.+n_{f}^{2} T_{f}^{2} \delta(1-z)\left(16-\frac{16}{9} \zeta(2)\right)\right) .
\end{aligned}
$$

The unphysical part is given by [see Eq. (2.35)]

$$
\begin{aligned}
& \hat{A}_{g g}^{\mathrm{EOM}}\left(z, \frac{-p^{2}}{\mu^{2}}, \frac{1}{\varepsilon}\right)=F\left[C_{A}\left(-2+8 z-8 z^{2}+\frac{1}{z}(1-\hat{\xi})\right)+\varepsilon C_{A}\left(-1+4 z-4 z^{2}+\frac{1}{2 z}(1-\hat{\xi})\right)[1+\ln (1-z)+\ln z]\right] \\
& +F^{2}\left\{\frac { 1 } { \varepsilon } \left[C_{A}^{2}\left(-\frac{184}{3}+\frac{6}{z}-\frac{80}{3} z+56 z^{2}-8 \ln z(1+16 z)-16 \ln (1-z)\left(1-4 z+4 z^{2}\right)\right)\right.\right. \\
& \left.+n_{f} C_{A} T_{f} \frac{8}{3}\left(4-\frac{1}{z}-16 z+16 z^{2}\right)+n_{f} C_{F} T_{f} \frac{64}{3}\left(3-\frac{1}{z}-2 z^{2}+3 z \ln z\right)\right] \\
& +C_{A}^{2}\left[\frac{2}{45}\left(-857+\frac{8}{z}-28 z+937 z^{2}\right)+\zeta(2)\left(10+\frac{8}{3 z}-25 z+8 z^{2}-\frac{248}{15} z^{3}\right)\right. \\
& +\zeta(3)\left(13-41 z+91 z^{2}-24 z^{3}\right)-4\left(1-4 z+4 z^{2}\right)\left\{3 \ln ^{2}(1-z)+\ln z \operatorname{Li}_{2}(-z)\right\}-\ln (1-z)\left(\frac{241}{3}\right. \\
& \left.-\frac{10}{3 z}-27 z-\frac{40}{3} z^{2}\right)+\ln (1-z) \zeta(2)\left(1+3 z-9 z^{2}+8 z^{3}\right)-\ln z \ln (1-z)\left(34+\frac{8}{3 z}+43 z+72 z^{2}\right) \\
& +\left(\frac{29}{3}+\frac{9}{5} \frac{1}{z^{2}}-4 z-32 z^{2}-\frac{248}{15} z^{3}\right)\left\{\ln z \ln (1+z)+\operatorname{Li}_{2}(-z)\right\}-2 \ln ^{2} z\left(3+47 z-\frac{62}{15} z^{3}\right) \\
& -2 \mathrm{Li}_{2}(1-z)\left(22+\frac{8}{3 z}+11 z+40 z^{2}\right)-\frac{1}{15} \ln z\left(829-\frac{8}{z}+2589 z-328 z^{2}\right) \\
& +\left(3-19 z+25 z^{2}-8 z^{3}\right)\left(-\ln (1-z) \operatorname{Li}_{2}(1-z)-\frac{1}{2} \ln ^{2} z \ln (1-z)-2 \ln z \operatorname{Li}_{2}(1-z)+\ln z \zeta(2)\right. \\
& \left.+3 \operatorname{Li}_{3}(1-z)\right)-\mathrm{S}_{12}(1-z)\left(13-73 z+91 z^{2}-24 z^{3}\right)+4 \operatorname{Li}_{3}(-z)\left(3-4 z+12 z^{2}\right)+4\left(1+4 z+4 z^{2}\right) \\
& \left.\times\left(-2 \ln (1+z) \mathrm{Li}_{2}(-z)-\ln (1+z) \zeta(2)-\ln z \ln ^{2}(1+z)+\frac{1}{2} \ln ^{2} z \ln (1+z)-2 \mathrm{~S}_{12}(-z)\right)\right] \\
& +n_{f} C_{A} T_{f}\left[\frac{88}{9}-\frac{28}{3 z}+\frac{128}{9} z-16 z^{2}+8 \ln (1-z)\left(\frac{7}{3}+\frac{1}{3 z}-6 z+4 z^{2}\right)+\frac{4}{3} \ln z\left(4-\frac{5}{z}-4 z+24 z^{2}\right)\right. \\
& +8\left(1+\frac{2}{3 z}-2 z\right)\left\{-\zeta(2)+\ln z \ln (1-z)+2 \mathrm{Li}_{2}(1-z)\right\} \\
& +16 z(1-z)\left(-\ln (1-z) \mathrm{Li}_{2}(1-z)-\ln (1-z) \zeta(2)-\frac{1}{2} \ln ^{2} z \ln (1-z)-2 \ln z \operatorname{Li}_{2}(1-z)+\ln z \zeta(2)\right.
\end{aligned}
$$




$$
\begin{aligned}
& \left.\left.+3 \zeta(3)+3 \mathrm{Li}_{3}(1-z)-3 \mathrm{~S}_{12}(1-z)\right)\right]+n_{f} C_{F} T_{f}\left[-\frac{64}{3}+\frac{128}{9 z}-\frac{32}{3} z+\frac{160}{9} z^{2}+64 \ln (1-z)\right. \\
& \left.\left.\times\left(1-\frac{1}{3 z}-\frac{2}{3} z^{2}\right)+32 \ln z\left(1+2 z-\frac{4}{3} z^{2}\right)+64 z\left(\frac{3}{4} \ln ^{2} z+\ln z \ln (1-z)+\operatorname{Li}_{2}(1-z)\right)\right]\right\} .
\end{aligned}
$$

The part which is due to the contribution of the NGI operators $O_{A}$ and $O_{\omega}$ [see Eq. (2.36)] is equal to

$$
\begin{aligned}
& \hat{A}_{g g}^{\mathrm{NGI}}\left(z, \frac{-p^{2}}{\mu^{2}}, \frac{1}{\varepsilon}\right)=F C_{A}\left[\frac{1}{\varepsilon}\left(4-\frac{4}{z}\right)+2\left(1-\frac{1}{z}\right)[1+\ln z+\ln (1-z)]+\varepsilon\left(1-\frac{1}{z}\right)\left(\frac{1}{2} \zeta(2)+\ln (1-z)+\ln z\right.\right. \\
& \left.\left.+\frac{1}{2}\{\ln z+\ln (1-z)\}^{2}\right)\right]+F^{2}\left(\frac { 1 } { \varepsilon ^ { 2 } } \left\{C _ { A } ^ { 2 } \left[\frac{4}{3}\left(1+2 z^{2}\right)+\frac{4}{z}-8 z+22 \ln (1-z)\left(1-\frac{1}{z}\right)+2 \ln z\right.\right.\right. \\
& \left.\left.\times\left(5+\frac{6}{z}\right)\right]-n_{f} C_{A} T_{f} \frac{32}{3}\left(1-\frac{1}{z}\right)\right\}+\frac{1}{\varepsilon}\left\{C _ { A } ^ { 2 } \left[-\frac{73}{9}+\frac{250}{9 z}-\frac{67}{3} z+\frac{8}{3} z^{2}+\frac{33}{2} \ln ^{2}(1-z)\left(1-\frac{1}{z}\right)\right.\right. \\
& +\frac{8}{3} \ln (1-z)\left(1+\frac{1}{z}-3 z+z^{2}\right)+2 \ln z \ln (1-z)\left(15-\frac{4}{z}\right)+\frac{1}{2} \ln ^{2} z\left(15+\frac{14}{z}\right) \\
& +\frac{2}{3} \ln z\left(65+\frac{18}{z}+6 z\right)+2 \operatorname{Li}_{2}(1-z)\left(14-\frac{3}{z}\right)+4\left(1+\frac{1}{z}\right)\left(\frac{1}{2} \zeta(2)+\ln z \ln (1+z)\right. \\
& \left.\left.\left.+\mathrm{Li}_{2}(-z)\right)\right]-n_{f} C_{A} T_{f} \frac{16}{3}\left(1-\frac{1}{z}\right)\left(\frac{1}{3}+2 \ln z+\ln (1-z)\right)\right\}+C_{A}^{2}\left[-\frac{59}{27}+\frac{1837}{54 z}-\frac{61}{2} z-\frac{4}{3} z^{2}\right. \\
& +\zeta(2)\left(\frac{1}{3}+\frac{2}{z}-5 z+\frac{2}{3} z^{2}\right)+\zeta(3)\left(5+\frac{2}{z}-18 z+9 z^{2}\right)+\frac{77}{12} \ln ^{3}(1-z)\left(1-\frac{1}{z}\right) \\
& +\ln ^{2}(1-z)\left(\frac{5}{3}+\frac{1}{z}-4 z+\frac{4}{3} z^{2}\right)+\ln (1-z) \operatorname{Li}_{2}(1-z)\left(29-\frac{2}{z}+6 z-3 z^{2}\right) \\
& +\frac{1}{9} \ln (1-z)\left(1+\frac{149}{z}-174 z+24 z^{2}\right)-\frac{1}{2} \ln (1-z) \zeta(2)\left(1+\frac{5}{z}-12 z+6 z^{2}\right) \\
& +\ln z \ln ^{2}(1-z)\left(22-\frac{7}{z}\right)+\frac{1}{3} \ln z \ln (1-z)\left(134+\frac{23}{z}+21 z\right)+\frac{1}{2} \ln ^{2} z \ln (1-z)\left(33-\frac{7}{z}+6 z\right. \\
& \left.-3 z^{2}\right)+\ln ^{3} z\left(\frac{35}{12}+\frac{5}{2 z}\right)+\ln ^{2} z\left(\frac{193}{6}+\frac{9}{z}+5 z-\frac{2}{3} z^{2}\right)+2 \ln z \operatorname{Li}_{2}(1-z)\left(13-\frac{7}{z}+6 z-3 z^{2}\right) \\
& +\ln z\left(\frac{985}{18}+\frac{412}{9 z}+\frac{71}{6} z\right)+\ln z \zeta(2)\left(\frac{9}{2}+\frac{4}{z}-6 z+3 z^{2}\right)+2 \operatorname{Li}_{2}(1-z)\left(22+\frac{4}{3 z}+7 z-\frac{2}{3} z^{2}\right) \\
& -\left(3-\frac{1}{z^{2}}+\frac{2}{z}\right)\left\{\ln z \ln (1+z)+\operatorname{Li}_{2}(-z)\right\}-\operatorname{Li}_{3}(1-z)\left(23-\frac{2}{z}+18 z-9 z^{2}\right) \\
& +S_{12}(1-z)\left(15-\frac{17}{z}+18 z-9 z^{2}\right)-2\left(1+\frac{1}{z}\right)\left(-\ln z \operatorname{Li}_{2}(-z)-2 \ln (1+z) \zeta(2)\right. \\
& -4 \ln (1+z) \operatorname{Li}_{2}(-z)-2 \ln (1+z) \operatorname{Li}_{2}(1-z)-2 \ln z \ln (1-z) \ln (1+z)-2 \ln z \ln ^{2}(1+z) \\
& \left.\left.-\frac{1}{2} \ln ^{2} z \ln (1+z)+\mathrm{Li}_{3}(-z)-6 \mathrm{~S}_{12}(-z)+\mathrm{S}_{12}\left(z^{2}\right)\right)\right]-n_{f} C_{A} T_{f} \frac{4}{3}\left(1-\frac{1}{z}\right) \\
& \left.\times\left(\frac{32}{9}+\frac{2}{3}\{\ln (1-z)+2 \ln z\}+2 \zeta(2)+\{2 \ln z+\ln (1-z)\}^{2}\right)\right) \text {. }
\end{aligned}
$$




\section{APPENDIX B}

In this appendix we present the non-gauge invariant (NGI) operators with their corresponding operator matrix elements (OME's). They are needed for the renormalization of the physical operators due to the mixing between them discussed in Sec. II. Two of them are given in Eqs. (2.9) and (2.10) of [15] (for their construction see [18]). These operators, which already show up in the case where only the gluonic operator in Eq. (2.3) is present, are given by

$$
\begin{aligned}
O_{A}^{\mu_{1}, \mu_{2} \cdots \mu_{n}}(x)= & i^{n-2} \mathcal{S}\left(F_{a, \alpha}^{\mu_{1}}(x) D^{\alpha} \partial^{\mu_{2} \cdots} \partial^{\mu_{n-1}} A_{a}^{\mu_{n}}(x)+i g f_{a b c} F_{a, \alpha}^{\mu_{1}}(x) \sum_{i=2}^{n-1} \kappa_{i} \partial^{\alpha}\left\{\left[\partial^{\left.\mu_{2} \cdots \partial^{\mu_{i-2}} A_{b}^{\mu_{i-1}}(x)\right]}\right.\right.\right. \\
& \left.\left.\times\left[\partial^{\mu_{i} \cdots} \partial^{\mu_{n-1-i}} A_{c}^{\mu_{n}}(x)\right]\right\}+\mathcal{O}\left(g^{2}\right)\right)
\end{aligned}
$$

and

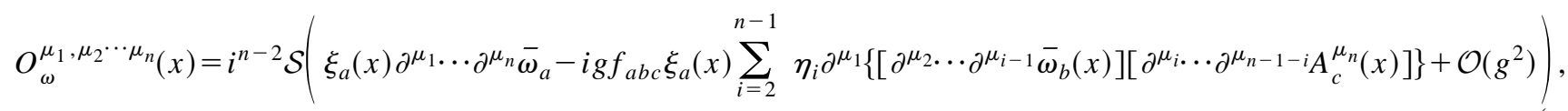

where $\xi$ and $\bar{\omega}$ are the ghost and antighost respectively. In the expressions above $\eta$ is defined in Eq. (2.45). Further $\kappa_{i}$ and $\eta_{i}$ are calculated in [15] and are given by

$$
\kappa_{i}=\frac{1}{8}(-1)^{i}+\frac{3}{8} \frac{(n-2) !}{(i-1) !(n-i-1) !}-\frac{3}{8} \frac{(n-2) !}{i !(n-i-2) !}
$$

and

$$
\eta_{i}=\frac{1}{4}(-1)^{i}+\frac{3}{4} \frac{(n-2) !}{(i-1) !(n-i-1) !}+\frac{1}{4} \frac{(n-2) !}{i !(n-i-2) !},
$$

respectively. If we also include the quark singlet operators in Eq. (2.2), then we have to add

$$
O_{B}^{\mu_{1}, \mu_{2} \cdots \mu_{n}}(x)=i^{n-1} \mathcal{S}\left[g \bar{\psi}_{k}(x) \gamma^{\mu_{1}}\left(T_{a}\right)_{k l} A_{a}^{\mu_{2}}(x) \partial^{\mu_{3} \cdots} \partial^{\mu_{n}} \psi_{l}(x)+\mathcal{O}\left(g^{3}\right)\right] \text {. }
$$

Notice that the above operators are not BRS exact in the strict sense of [17] (see also [21]). This might affect the nonlogarithmic terms in the renormalized OME's which we do not need here. Further the operators in the above equations are corrected up to order $g^{2}$. This is sufficient to get finite two-loop OME's. However, in order to carry out the renormalization on the three-loop level one has to compute higher order corrections to Eqs. (B1), (B2) and (B5). The operator vertices corresponding to $O_{A}$ in Eq. (B1) and $O_{B}$ in Eq. (B2), albeit sandwiched between gluon states, are presented in Appendix A.3 of [15]. In Sec. II we need the $O_{B}$ operator vertex when it is sandwiched between quark states. In this case we have to compute the quark-quark-gluon vertex which is given by

$$
V_{a, k l}^{\mu}=g\left(T_{a}\right)_{k l} \Delta / \Delta^{\mu}(\Delta \cdot k)^{n-2},
$$

where $k$ stands for the momentum of the gluon.

We now list the OME's needed for the renormalization of the physical operators in Sec. II (for the notation see Appendix A). The two OME's referring to the NGI operators $O_{A}$ and $O_{B}$, when sandwiched between quarks states, can be split according to Eq. (2.7). The expressions in Eqs. (2.46) and (2.47) become

$$
\begin{aligned}
& \hat{A}_{A q}^{\mathrm{PHYS}}\left(z, \frac{-p^{2}}{\mu^{2}}, \frac{1}{\varepsilon}\right) \\
&= F C_{F}\left(\frac{1}{\varepsilon}\left(-8+8 \frac{1}{z}\right)-2+2 \frac{1}{z}+\left(-4+4 \frac{1}{z}\right)[\ln (1-z)+\ln z]-(1-\hat{\xi})\left(2-\frac{3}{2 z}\right)\right. \\
&-\varepsilon\left\{\left(1-\frac{1}{z}\right)\left[\ln (1-z)+\ln z+\{\ln (1-z)+\ln z\}^{2}+\zeta(2)\right]+(1-\hat{\xi})\left[\frac{1}{2}-\frac{1}{4 z}+\left(1-\frac{3}{4 z}\right)\{\ln (1-z)+\ln z\}\right]\right\}
\end{aligned}
$$

and

$$
\hat{A}_{A q}^{\mathrm{EOM}}\left(z, \frac{-p^{2}}{\mu^{2}}, \frac{1}{\varepsilon}\right)=(1-\hat{\xi}) F C_{F}\left(1+\frac{\varepsilon}{2}[1+\ln (1-z)+\ln z]\right),
$$


respectively. Likewise we obtain for Eqs. (2.48) and (2.49)

$$
\begin{aligned}
\hat{A}_{B q}^{\mathrm{PHYS}}\left(z, \frac{-p^{2}}{\mu^{2}}, \frac{1}{\varepsilon}\right)= & F C_{F}\left[\frac{1}{\varepsilon}\left(8-8 \frac{1}{z}\right)+\left(4-4 \frac{1}{z}\right)[\ln (1-z)+\ln z]+(1-\hat{\xi})\left(2-\frac{2}{z}\right)\right. \\
& \left.+\varepsilon\left(1-\frac{1}{z}\right)\left\{[\ln (1-z)+\ln z]^{2}+\zeta(2)+(1-\hat{\xi})[\ln (1-z)+\ln z]\right\}\right]
\end{aligned}
$$

and

$$
\hat{A}_{B q}^{\mathrm{EOM}}\left(z, \frac{-p^{2}}{\mu^{2}}, \frac{1}{\varepsilon}\right)=0,
$$

respectively. Next we sandwich the NGI operators $O_{A}$ and $O_{\omega}$ between gluon states. Decomposing the OME's according to Eq. (2.53) we get for $\hat{A}_{A g}$ the following results. The physical and unphysical parts [see Eqs. (2.54) and (2.55)] become equal to

$$
\begin{aligned}
\hat{A}_{A g}^{\mathrm{PHYS}}\left(z, \frac{-p^{2}}{\mu^{2}}, \frac{1}{\varepsilon}\right)= & F C_{A}\left(\frac{1}{\varepsilon}\left[2 z-2 z^{2}+(1-\hat{\xi})\left(12-\frac{2}{z}-3 z-2 z^{2}\right)+(1-\hat{\xi})^{2}\left(\frac{1}{2}-z-\frac{z^{2}}{2}\right)\right]+z(1-z)[\ln (1-z)+\ln z]\right. \\
& +2-4 \frac{1}{z}+(1-\hat{\xi})\left[\frac{7}{2}+\frac{1}{z^{2}}-\frac{5}{2 z}-\frac{z}{2}+\left(6-\frac{1}{z}-\frac{3}{2} z-z^{2}\right)\{\ln (1-z)+\ln z\}\right] \\
& +(1-\hat{\xi})^{2}\left(\frac{7}{8}-\frac{3}{4} z+\frac{1}{4}\left(1-2 z-z^{2}\right)\{\ln (1-z)+\ln z\}\right)+\varepsilon\left\{\left(1-\frac{2}{z}\right)[\ln (1-z)+\ln z]\right. \\
& +\frac{1}{4} z(1-z)\left[\{\ln (1-z)+\ln z\}^{2}+\zeta(2)\right]+(1-\hat{\xi})\left[-\frac{1}{2 z}+\left(\frac{7}{4}+\frac{1}{2 z^{2}}-\frac{5}{4 z}-\frac{1}{4} z\right)\{\ln (1-z)+\ln z\}\right. \\
& \left.+\left(\frac{3}{2}-\frac{1}{4 z}-\frac{3 z}{8}-\frac{z^{2}}{4}\right)\left\{[\ln (1-z)+\ln z]^{2}+\zeta(2)\right\}\right]+(1-\hat{\xi})^{2}\left[\frac{1}{16}+\left(\frac{7}{16}-\frac{3}{8} z\right)\{\ln (1-z)+\ln z\}\right. \\
& \left.\left.\left.+\frac{1}{16}\left(1-2 z-z^{2}\right)\left\{[\ln (1-z)+\ln z]^{2}+\zeta(2)\right\}\right]\right\}\right)
\end{aligned}
$$

and

$$
\begin{aligned}
\hat{A}_{A g}^{\mathrm{EOM}}\left(z, \frac{-p^{2}}{\mu^{2}}, \frac{1}{\varepsilon}\right)= & F C_{A}\left(-\frac{1}{\varepsilon} 13 \delta(1-z)(1-\hat{\xi})+4+2 z-2 z^{2}+(1-\hat{\xi})\left(5-\frac{1}{z^{2}}+\frac{5}{2 z}-2 z-2 z^{2}+\delta(1-z)\right.\right. \\
& \left.-\frac{1}{2} \frac{1}{1-z}-\frac{3}{2} \frac{1}{(1-z)^{2}}\right)+(1-\hat{\xi})^{2}\left(\frac{3}{2}-\frac{1}{2 z}-2 z-\frac{z^{2}}{2}-\frac{27}{8} \delta(1-z)-\frac{3}{4} \frac{1}{1-z}\right) \\
& +\varepsilon\left\{\left(2+z-z^{2}\right)[\ln (1-z)+\ln z]+(1-\hat{\xi})\left[\frac{5}{2}-\frac{z}{2}-\frac{1}{1-z}+\delta(1-z)\left\{-\frac{15}{2}+\frac{11}{8} \zeta(2)\right\}\right.\right. \\
& \left.+\left(\frac{5}{2}-\frac{1}{2 z^{2}}+\frac{5}{4 z}-z-z^{2}-\frac{1}{4} \frac{1}{1-z}-\frac{3}{4} \frac{1}{(1-z)^{2}}\right)\{\ln (1-z)+\ln z\}\right]+(1-\hat{\xi})^{2}\left[\frac{3}{4}-\frac{1}{8 z^{2}}+\frac{3}{16 z}-\frac{3 z}{4}\right. \\
& \left.\left.\left.+\frac{3}{2} \delta(1-z)\left(1-\frac{1}{4} \zeta(2)\right)-\frac{3}{16} \frac{1}{(1-z)^{2}}-\frac{3}{8} \frac{1}{1-z}+\left(\frac{3}{4}-\frac{1}{4 z}-z-\frac{z^{2}}{4}-\frac{3}{8} \frac{1}{1-z}\right)\{\ln (1-z)+\ln z\}\right]\right\}\right),
\end{aligned}
$$

respectively. The piece coming from the NGI operator $O_{A}$ itself and the part due to the violation of the WI [see Eqs. (2.56), (2.57)] are given by

$$
\begin{aligned}
\hat{A}_{A g}^{\mathrm{NGI}}\left(z, \frac{-p^{2}}{\mu^{2}}, \frac{1}{\varepsilon}\right)= & F C_{A}\left(\frac { 1 } { \varepsilon } \left[2-5 z+2 z^{2}+\frac{4}{z}-6 \frac{1}{1-z}-\frac{3}{2} \delta(1-z)+(1-\hat{\xi})\left(\frac{61}{2}-\frac{21}{2 z}-\frac{21 z}{2}-z^{2}+\frac{145}{24} \delta(1-z)+\frac{5}{4} \frac{1}{1-z}\right)\right.\right. \\
& \left.+(1-\hat{\xi})^{2}\left(-\frac{3}{2}-\frac{1}{2 z}+\frac{11 z}{4}-\frac{5}{2} z^{2}+\frac{11}{12} \delta(1-z)-\frac{3}{4} \frac{1}{1-z}\right)\right]+\left(1-\frac{5}{2} z+z^{2}+\frac{2}{z}-\frac{3}{1-z}\right)[\ln (1-z)
\end{aligned}
$$




$$
\begin{aligned}
& +\ln z]-\frac{5}{2}+\frac{2}{z}+\delta(1-z)\left(\frac{4}{3}-3 \zeta(2)\right)+(1-\hat{\xi})\left[\frac{17}{2}+\frac{1}{8 z^{2}}-\frac{41}{16 z}-3 z+\delta(1-z)\left(-\frac{1}{72}+\frac{5}{8} \zeta(2)\right)\right. \\
& \left.+\frac{7}{16} \frac{1}{1-z}+\frac{3}{16} \frac{1}{(1-z)^{2}}+\left(\frac{61}{4}-\frac{21}{4 z}-\frac{21 z}{4}-\frac{z^{2}}{2}+\frac{5}{8} \frac{1}{1-z}\right)\{\ln (1-z)+\ln z\}\right]+(1-\hat{\xi})^{2}\left[\frac{7}{4}+\frac{1}{8 z^{2}}\right. \\
& -\frac{15}{16 z}-\frac{1}{2} z+\delta(1-z)\left(\frac{97}{36}-\frac{3}{8} \zeta(2)\right)+\frac{11}{16} \frac{1}{1-z}+\frac{3}{16} \frac{1}{(1-z)^{2}}+\left(-\frac{3}{4}-\frac{1}{4 z}+\frac{11}{8} z-\frac{5}{4} z^{2}-\frac{3}{8} \frac{1}{1-z}\right) \\
& \times\{\ln (1-z)+\ln z\}]+\varepsilon\left\{\delta(1-z)\left(-\frac{23}{18}+\frac{3}{16} \zeta(2)+3 \zeta(3)\right)+\left(-\frac{5}{4}+\frac{1}{z}\right)[\ln (1-z)+\ln z]+\frac{1}{8}(2\right. \\
& \left.+\frac{4}{z}-5 z+2 z^{2}-\frac{3}{4} \frac{1}{1-z}\right)\left[\{\ln (1-z)+\ln z\}^{2}+\zeta(2)\right]+(1-\hat{\xi})\left[-\frac{1}{2}+\frac{1}{8 z}+\frac{7}{32} \frac{\ln z}{1-z}+\frac{7}{32} \frac{\ln (1-z)}{1-z}\right. \\
& +\frac{1}{2} \frac{1}{1-z} \\
& +\delta(1-z)\left(\frac{677}{432}-\frac{103}{192} \zeta(2)-\frac{5}{8} \zeta(3)\right)+\left(\frac{17}{4}+\frac{1}{16 z^{2}}-\frac{41}{32 z}-\frac{3}{2} z+\frac{3}{32} \frac{1}{(1-z)^{2}}\right)[\ln (1-z)+\ln z] \\
& \left.+\frac{1}{16}\left(61-\frac{21}{z}-21 z-2 z^{2}+\frac{5}{2} \frac{1}{1-z}\right)\left[\{\ln z+\ln (1-z)\}^{2}+\zeta(2)\right]\right]+(1-\hat{\xi})^{2}\left[\frac{5}{16}+\frac{1}{16 z^{2}}-\frac{5}{16 z}\right. \\
& +\frac{3}{32} \frac{1}{(1-z)^{2}}+\frac{11}{32} \frac{\ln z}{1-z}+\frac{11}{32} \frac{\ln (1-z)}{1-z}+\frac{15}{32} \frac{1}{1-z}+\delta(1-z)\left(-\frac{271}{432}+\frac{11}{48} \zeta(2)+\frac{3}{8} \zeta(3)\right) \\
& +\left(\frac{7}{8}+\frac{1}{16 z^{2}}-\frac{15}{32 z}-\frac{1}{4} z+\frac{3}{32} \frac{1}{(1-z)^{2}}\right)\{\ln (1-z)+\ln z\}+\frac{1}{16}\left(-3-\frac{1}{z}+\frac{11}{2} z\right. \\
& \left.\left.\left.\left.-5 z^{2}-\frac{3}{2} \frac{1}{1-z}\right)\left[\{\ln (1-z)+\ln z\}^{2}+\zeta(2)\right]\right]\right\}\right)
\end{aligned}
$$

and

$$
\begin{aligned}
\hat{A}_{A g}^{\mathrm{WI}}\left(z, \frac{-p^{2}}{\mu^{2}}, \frac{1}{\varepsilon}\right)= & F C_{A}\left(\frac{1}{\varepsilon}\left[(1-\hat{\xi})\left(5-z-\frac{2}{z}-\frac{5}{8} \delta(1-z)\right)+(1-\hat{\xi})^{2}\left(-4+\frac{5}{2 z}+z+\frac{1}{4} \delta(1-z)+\frac{1}{4} \frac{1}{1-z}\right)\right]+\frac{1}{2}+(1-\hat{\xi})\right. \\
& \times\left[\frac{1}{4}-\frac{1}{8 z^{2}}-\frac{11}{16 z}+\frac{3}{8} \delta(1-z)+\frac{7}{16} \frac{1}{1-z}-\frac{3}{16} \frac{1}{(1-z)^{2}}+\left(\frac{5}{2}-\frac{1}{z}-\frac{z}{2}\right)\{\ln (1-z)+\ln z\}\right]+(1-\hat{\xi})^{2} \\
& \times\left[-\frac{5}{8}+\frac{1}{z}+\delta(1-z)\left(-\frac{9}{16}+\frac{1}{8} \zeta(2)\right)-\frac{1}{16} \frac{1}{1-z}+\left(-2+\frac{5}{4 z}+\frac{z}{2}+\frac{1}{8} \frac{1}{1-z}\right)\{\ln (1-z)+\ln z\}\right] \\
& +\varepsilon\left\{\frac{1}{4}[\ln (1-z)+\ln z]+(1-\hat{\xi})\left[-\frac{1}{8 z}+\frac{7}{32} \frac{\ln z}{1-z}+\frac{7}{32} \frac{\ln (1-z)}{1-z}+\delta(1-z)\left(-\frac{15}{16}+\frac{19}{64} \zeta(2)\right)\right.\right. \\
& \left.+\left(\frac{1}{8}-\frac{1}{16 z^{2}}-\frac{11}{32 z}-\frac{3}{32} \frac{1}{(1-z)^{2}}\right)\{\ln z+\ln (1-z)\}+\frac{1}{8}\left(5-\frac{2}{z}-z\right)\left\{[\ln (1-z)+\ln z]^{2}+\zeta(2)\right\}\right] \\
& +(1-\hat{\xi})^{2}\left[\frac{1}{32 z}-\frac{3}{32} \frac{1}{1-z}-\frac{1}{32} \frac{\ln z}{1-z}-\frac{1}{32} \frac{\ln (1-z)}{1-z}+\delta(1-z)\left(\frac{3}{8}-\frac{1}{16} \zeta(2)-\frac{1}{8} \zeta(3)\right)\right. \\
& \left.\left.\left.+\left(-\frac{5}{16}+\frac{1}{2 z}\right)\{\ln (1-z)+\ln z\}+\frac{1}{16}\left(-8+\frac{5}{z}+2 z+\frac{1}{2} \frac{1}{1-z}\right)\left[\{\ln (1-z)+\ln z\}^{2}+\zeta(2)\right]\right\}\right\}\right)
\end{aligned}
$$

respectively.

The last OME due to $O_{\omega}$ can be also split into four pieces according to Eq. (2.58). For Eq. (2.59) we get

$$
\hat{A}_{\omega g}^{\mathrm{PHYS}}\left(z, \frac{-p^{2}}{\mu^{2}}, \frac{1}{\varepsilon}\right)=-F C_{A} z(1-z)\left(\frac{2}{\varepsilon}+\ln (1-z)+\ln z+\varepsilon \frac{1}{4}\left[\{\ln (1-z)+\ln z\}^{2}+\zeta(2)\right]\right) .
$$


The expression in Eq. (2.60) becomes

$$
\hat{A}_{\omega g}^{\mathrm{EOM}}\left(z, \frac{-p^{2}}{\mu^{2}}, \frac{1}{\varepsilon}\right)=-F C_{A} z(1-z)\{2+\varepsilon[\ln (1-z)+\ln z]\} .
$$

For the contribution due to the NGI operator $O_{\omega}$ we get [see Eq. (2.61)]

$$
\begin{aligned}
\hat{A}_{\omega g}^{\mathrm{NGI}}\left(z, \frac{-p^{2}}{\mu^{2}}, \frac{1}{\varepsilon}\right)= & F C_{A}\left\{\frac{1}{\varepsilon}\left(-2+5 z-2 z^{2}+\frac{1}{6} \delta(1-z)\right)+\left(-1+\frac{5}{2} z-z^{2}\right)[\ln (1-z)+\ln z]+\frac{1}{2}-\frac{2}{9} \delta(1-z)\right. \\
& \left.+\varepsilon\left[\delta(1-z)\left(\frac{13}{54}-\frac{1}{48} \zeta(2)\right)+\frac{1}{4}[\ln (1-z)+\ln z]+\frac{1}{8}\left(-2+5 z-2 z^{2}\right)\left[\{\ln (1-z)+\ln z\}^{2}+\zeta(2)\right]\right]\right\} .
\end{aligned}
$$

Finally the contribution due to the breakdown of the Ward identity is [see Eq. (2.62)]

$$
\hat{A}_{\omega g}^{\mathrm{WI}}\left(z, \frac{-p^{2}}{\mu^{2}}, \frac{1}{\varepsilon}\right)=-F C_{A}\left(\frac{1}{2}+\varepsilon \frac{1}{4}[\ln (1-z)+\ln z]\right) .
$$

[1] H. D. Politzer, Phys. Rep. 14, 129 (1974); A. J. Buras, Rev. Mod. Phys. 52, 199 (1980); G. Altarelli, Phys. Rep. 81, 1 (1982).

[2] G. Sterman et al., Rev. Mod. Phys. 67, 157 (1995).

[3] H. Georgi and H. D. Politzer, Phys. Rev. D 9, 416 (1974).

[4] D. J. Gross and F. Wilczek, Phys. Rev. D 9, 980 (1974).

[5] G. Altarelli and G. Parisi, Nucl. Phys. B126, 298 (1977).

[6] E. G. Floratos, D. A. Ross and C. T. Sachrajda, Nucl. Phys. B129, 66 (1977); B139, 545(E) (1978); B152, 493 (1979).

[7] A. Gonzales-Arroyo, C. Lopez and F. J. Yndurain, Nucl. Phys. B153, 161 (1979); A. Gonzalez-Arroyo and C. Lopez, ibid. B166, 429 (1980).

[8] G. Curci, W. Furmanski and R. Petronzio, Nucl. Phys. B175, 27 (1980); W. Furmanski and R. Petronzio, Phys. Lett. 97B, 437 (1980); Z. Phys. C 11, 293 (1982).

[9] E. G. Floratos, C. Kounnas and R. Lacaze, Phys. Lett. 98B, 89 (1981); 98B, 285 (1981); Nucl. Phys. B192, 417 (1981).

[10] Proceedings of the workshop 1995/96 "Future Physics at HERA,' edited by G. Ingelman, A. de Roeck and R. Klanner, DESY, Hamburg, 1996 (unpublished), Vols. 1 and 2.

[11] E. B. Zijlstra and W. L. van Neerven, Nucl. Phys. B383, 525 (1992); Phys. Lett. B 297, 377 (1992).

[12] R. Hamberg, W. L. van Neerven and T. Matsuura, Nucl. Phys. B359, 343 (1991); W. L. van Neerven and E. B. Zijlstra, ibid. B382, 11 (1992).

[13] S. A. Larin, T. van Ritbergen and J. A. M. Vermaseren, Nucl. Phys. B427, 41 (1994); S. A. Larin, P. Nogueira, T. van Rit- bergen and J. A. M. Vermaseren, ibid. B492, 338 (1997); See also J. F. Bennett and J. A. Gracey, hep-ph/9710364.

[14] A. L. Kataev, A. V. Kotikov, C. Parente and A. V. Sidorov, Phys. Lett. B 388, 179 (1996).

[15] R. Hamberg and W. L. van Neerven, Nucl. Phys. B379, 143 (1992).

[16] R. Hamberg, Ph.D. thesis, University of Leiden, 1991.

[17] C. Becchi, A. Rouet and R. Stora, Phys. Lett. 52B, 344 (1974); Commun. Math. Phys. 42, 127 (1975); Ann. Phys. (N.Y.) 98, 287 (1976).

[18] J. A. Dixon and J. C. Taylor, Nucl. Phys. B78, 552 (1974).

[19] H. Kluberg-Stern and J. B. Zuber, Phys. Rev. D 12, 467 (1975); 12, 3159 (1975).

[20] S. D. Joglekar and B. W. Lee, Ann. Phys. (N.Y.) 97, 160 (1976); S. D. Joglekar, ibid. 108, 233 (1977); 109, 210 (1977).

[21] J. C. Collins and R. J. Scalise, Phys. Rev. D 50, 4117 (1994).

[22] B. W. Harris and J. Smith, Phys. Rev. D 51, 4550 (1995).

[23] M. Buza, Y. Matiounine, J. Smith, R. Migneron and W. L. van Neerven, Nucl. Phys. B472, 611 (1996).

[24] J. A. M. Vermaseren, FORM2, published by Computer Algebra Netherlands (CAN), Kruislaan 413, 1098SJ Amsterdam, The Netherlands.

[25] S. L. Adler, Physica A 143, 1144 (1966).

[26] L. Lewin, Polylogarithms and Associated Functions (NorthHolland, Amsterdam, 1983); R. Barbieri, J. A. Mignaco and E. Remiddi, Nuovo Cimento A 11, 824 (1972); A. Devoto and D. W. Duke, Riv. Nuovo Cimento 7, 1 (1984). 